\title{
Dendritic cell dysfunction and diabetic sensory neuropathy in the cornea
}

\author{
Nan Gao, ${ }^{1}$ Chenxi Yan, ${ }^{1,2}$ Patrick Lee, ${ }^{1}$ Haijing Sun, ${ }^{1}$ and Fu-Shin Yu ${ }^{1}$ \\ 'Departments of Ophthalmology, Anatomy and Cell Biology, Wayne State University School of Medicine, Detroit, Michigan, USA. Department of Ophthalmology, Shanghai Ninth Peoples' Hospital, \\ Shanghai Jiao Tong University School of Medicine, Shanghai, China.
}

\begin{abstract}
Diabetic peripheral neuropathy (DPN) often leads to neurotrophic ulcerations in the cornea and skin; however, the underlying cellular mechanisms of this complication are poorly understood. Here, we used post-wound corneal sensory degeneration and regeneration as a model and tested the hypothesis that diabetes adversely affects DC populations and infiltration, resulting in disrupted DC-nerve communication and DPN. In streptozotocin-induced type 1 diabetic mice, there was a substantial reduction in sensory nerve density and the number of intraepithelial DCs in unwounded (UW) corneas. In wounded corneas, diabetes markedly delayed sensory nerve regeneration and reduced the number of infiltrating DCs, which were a major source of ciliary neurotrophic factor (CNTF) in the cornea. While CNTF neutralization retarded reinnervation in normal corneas, exogenous CNTF accelerated nerve regeneration in the wounded corneas of diabetic mice and healthy animals, in which DCs had been locally depleted. Moreover, blockade of the CNTF-specific receptor CNTFR $\alpha$ induced sensory nerve degeneration and retarded regeneration in normal corneas. Soluble CNTFR $\alpha$ also partially restored the branching of diabetes-suppressed sensory nerve endings and regeneration in the diabetic corneas. Collectively, our data show that DCs mediate sensory nerve innervation and regeneration through CNTF and that diabetes reduces DC populations in UW and wounded corneas, resulting in decreased CNTF and impaired sensory nerve innervation and regeneration.
\end{abstract}

\section{Introduction}

The cornea is primarily innervated by small-diameter C-fiber sensory nerves and is the most densely innervated mammalian tissue (1). Axons from the trigeminal ganglion terminate in delicate endings among the epithelial cells of the cornea (2). The sensory nerves are responsible for the sensations of dryness, temperature, touch, and pain and play important roles in the blink reflex, wound healing, and tear production (3). Many ocular and systemic diseases can adversely affect corneal sensory nerves, resulting in neurotrophic keratopathy and even severe ulceration (4-6). One of these systemic diseases is diabetes mellitus (DM), which causes sensory nerve degeneration, termed diabetic neurotrophic keratopathy (DNK), and delayed wound healing (7-9). DNK is a complication of DM that is difficult to manage $(7,10,11)$. The mechanisms underlying the pathogenesis of DNK largely remain to be determined.

Diabetic peripheral neuropathy (DPN) is a common and debilitating complication, with more than half of all patients developing altered sensation as a result of damage to peripheral sensory nerves, which are known to be preferentially affected in the early stages of diabetes (12-15). In the cornea, a decrease in the density of sensory nerves occurs during the early stages of diabetes $(8,9$, $16-18)$, and this nerve damage is known to be directly related to the severity of somatic neuropathy in diabetic patients $(3,19,20)$. To date, the underlying basis for nerve damage is not well established, although the reduction or loss of neurotrophic signals has been implicated $(21,22)$. Our previous studies using rats and

Conflict of interest: The authors have declared that no conflict of interest exists. Submitted: October 13, 2015; Accepted: February 24, 2016.

Reference information: J Clin Invest. 2016;126(5):1998-2011. doi:10.1172/JCI85097. mice with streptozotocin-induced type $1 \mathrm{DM}$ (STZ type $1 \mathrm{DM})$ and rats with Goto-Kakizaki type $2 \mathrm{DM}$ (GK type $2 \mathrm{DM}$ ) revealed that defects in the sensory nerves contribute to DNK and delayed epithelial wound healing $(8,9)$. The cornea is easily accessible and has a battery of clinical noninvasive tests that can be adapted to animal models $(8,18,23)$. We and others have adapted these tests and documented a correlation between the severity of diabetic sensory neuropathy and the duration of diabetes in rat corneas $(8,9,17,18,24)$. Using the cornea as the experimental model, we sought to explore the mechanisms underlying DPN.

Neuro-immune interactions have attracted much attention recently, and DCs are at the center of these interactions (25-29). A 2009 study revealed that DCs were in contact with sensory nerve endings and T cells and that nerve-contacting DCs induced $\mathrm{T}$ cell proliferation in the airways of mice with allergic inflammation (30). More recently, it was reported that DCs interact with sensory nerves during allergic airway inflammation in a calcitonin gene-related peptide-related (CGRP-related) manner (31). In the cornea, an accumulation of DCs was found to be associated with nerve fiber damage of the sub-basal nerve plexus (32). We previously showed that DCs are required for proper wound healing in the cornea and, more recently, that DCs and sensory nerves are structurally connected and functionally interdependent (33). While sensory nerve influences on DCs have been documented (34-36), to date, understanding how DCs influence sensory nerve structure and function in tissue homeostasis and in response to environmental challenges, such as wounding and/ or diabetes, remains elusive.

In the present study, we focused on the functional interactions of these 2 cells and showed that sensory nerve density and 
DC populations were dramatically decreased in DM corneas. We also showed, for the first time to our knowledge, that ciliary neurotrophic factor (CNTF), a major neurotrophic factor (NTF) involved in sensory nerve survival and regeneration, originates from DCs; a decrease in the DC population during wound healing resulted in the reduction of tissue levels of CNTF, which in turn hindered sensory nerve regeneration. Our results will not only help to increase our understanding of DPN but also to potentially identify therapeutics for the treatment of diabetic neurotrophic ulcerations in the cornea and skin.

\section{Results}

Diabetes decreases the density of sensory nerve fibers and endings and the number ofCD11c-positive cells in corneas. Eight-week-old female B6 mice were induced to develop diabetes using the low-dose STZ induction protocol, with $0.1 \mathrm{M}$ citrate buffer $(\mathrm{pH} 4.5)$ as the control. Average blood sugar levels reached $300 \mathrm{dg} / \mathrm{ml}$ by week 2 and were $350-500 \mathrm{dg} / \mathrm{ml}$ by week 10 , after completion of the STZ regimen, while the control mice had average blood sugar levels of approximately $100 \mathrm{dg} / \mathrm{ml}$. Our previous studies showed that at this time point or thereafter, epithelial wound healing was significantly delayed in the corneas of STZ type $1 \mathrm{DM}$ mice compared with that seen in citrate buffer-injected normoglycemic (NL) mice (37) (see also Supplemental Figure 1; supplemental material available online with this article; doi:10.1172/JCI85097DS1).

We performed whole-mount confocal microscopic (WMCM) studies of DM and age-matched NL B6 mouse corneas to quantitatively determine whether diabetes adversely affects sensory regeneration and DC infiltration during epithelial wound healing. Figure 1 shows corneal innervation in NL and DM corneas before and after epithelial debridement. We defined the corneal fibers as being derived from the limbal nerve fibers and traveling through the stroma, with at least 1 branch; endings derived from corneal fibers were defined as the smallest unbranching fibers that run as a group and radially toward the whorl. These endings are located in the sub-basal nerve plexus. For unwounded (UW) corneas, 3 parameters were assessed: the total number of branches of the major nerve fibers (arrowheads), the length of these nerve fibers (between 2 arrows), and the density of the nerve endings at the center of the cornea (enlarged images, middle panel) (Figure 1A). In the UW corneas, the average length of the major nerve fibers, defined as starting from the beginning of a corneal fiber that branched perpendicularly from a larger limbal nerve to the distinguishable end of that corneal fiber, was $10,792 \mu \mathrm{m} \pm 1,214 \mu \mathrm{m}$ for NL corneas and $5,033 \mu \mathrm{m} \pm 1,002 \mu \mathrm{m}$ for DM corneas (Figure 1B); there were $28 \pm$ 7.6 branches of the major nerve fibers in NL corneas and $12 \pm 0.58$ branches in DM corneas (Figure 1C); and the density of sensory nerve fibers and endings at the center of the cornea, as measured by the $\beta$-tubulin III-stained area, was $19.7 \% \pm 4 \%$ in NL corneas and $9.1 \% \pm 0.59 \%$ in DM corneas (Figure 1D). For healing corneas, 18 and 24 hours post wounding (hpw) were chosen, since, at these time points, the sizes of the remaining wounds were similar (Supplemental Figure 1A). The percentages of areas that stained positive for $\beta$-tubulin III were $5.25 \% \pm 0.134 \%$ in NL corneas and $1.42 \%$ $\pm 0.295 \%$ in DM corneas (Figure 1E). Hence, both innervation in the UW corneas and sensory nerve regeneration in the healing corneas were significantly and adversely affected by diabetes.
The number of DCs in UW corneas was also quantitated using WMCM (Figures 2 and 3). We identified 2 morphologically different DC populations in the UW corneas: dendriform, the typical residential DC morphology, and round-shaped DCs, which were differently associated with sensory nerve fibers and endings. There were $130 \pm 13$ and $61 \pm 3$ intraepithelial CD11c-positive DCs in NL and DM corneas, respectively (Figure 2A), with both dendriform (arrows) and round-shaped (arrowheads) DCs counted (bottom panels, Figure 2A and B). While CD11c is considered the hallmark surface marker for the identification of DCs, MHC class II is another commonly used DC marker. To further confirm the identity of these CD11cpositive cells, we performed WMCM costaining of CD11c and MHC class II molecules and found that both types of DCs, dendriform (arrows) and round shaped, were also MHC class II positive in NL and DM mouse corneas (Figure 2C).

We previously showed that infiltrated DCs migrate along with the epithelial sheet to cover the wound (33). To assess the effects of diabetes on DC infiltration into wounded corneas, CD11c and MHC class II staining was done at $22 \mathrm{hpw}$, a time point at which the wounds in the NL corneas were nearly healed, whereas the wounds in the DM corneas remained relatively large (Supplemental Figure 1B). In wounded corneas, compared with UW corneas, much higher densities of CD11c- and MHC class II-positive cells were detected, and all CD11c- and/or MHC class II-positive cells were round shaped. Interestingly, unlike in UW corneas, while all CD11c-positive cells were also MHC class II positive, many MHC class II-positive cells were CD11c negative (Figure $3 \mathrm{~A}$ ). We detected markedly higher numbers of CD11c-positive cells in NL healing corneas than in DM corneas and moderate, but significant, differences in the numbers of MHC class II-positive cells (Figure 3B). We observed a larger number of CD11c-positive cells in NL corneas than in DM corneas 2 and 4 days post wounding (dpw), with the total number of cells declining rapidly over time (Supplemental Figure 2).

Nerve-DC interactions are interrupted during epithelial wound healing in DM corneas. We next used WMCM to investigate nerve-DC colocalization in UW and wounded corneas of NL and DM mice (Figure 4). In the peripheral region, we observed numerous dendriform (arrows) and round-shaped (arrowheads) DCs located near the limbus, with many nerve fiber branching points in NL corneas (Figure 4A). In DM corneas, we detected a few round-shaped and dendriform DCs from which endings were branching.

Figure 4B shows the colocalization of sensory nerves and DCs during epithelial wound healing. In NL healing corneas, epithelial innervation was robust, and the regenerating nerve fibers were frequently branching to form nerve endings. These newly grown fibers were uniform in size and evenly distributed, with numerous DCs in the region behind the leading edge of the migratory epithelial sheets (NL1, Figure 4B). In the DM corneas, fewer nerve insertion sites were observed near the limbus, and there was less density of regenerating nerve fibers than that seen in the controls. Strikingly, these nerve fibers were tortuous, discontinuous, or fragmented, similar to that observed in diabetic human corneas (38). We found few DCs in the region behind the leading edge (DM1, Figure 4B) and markedly higher numbers of DCs near the leading 
A
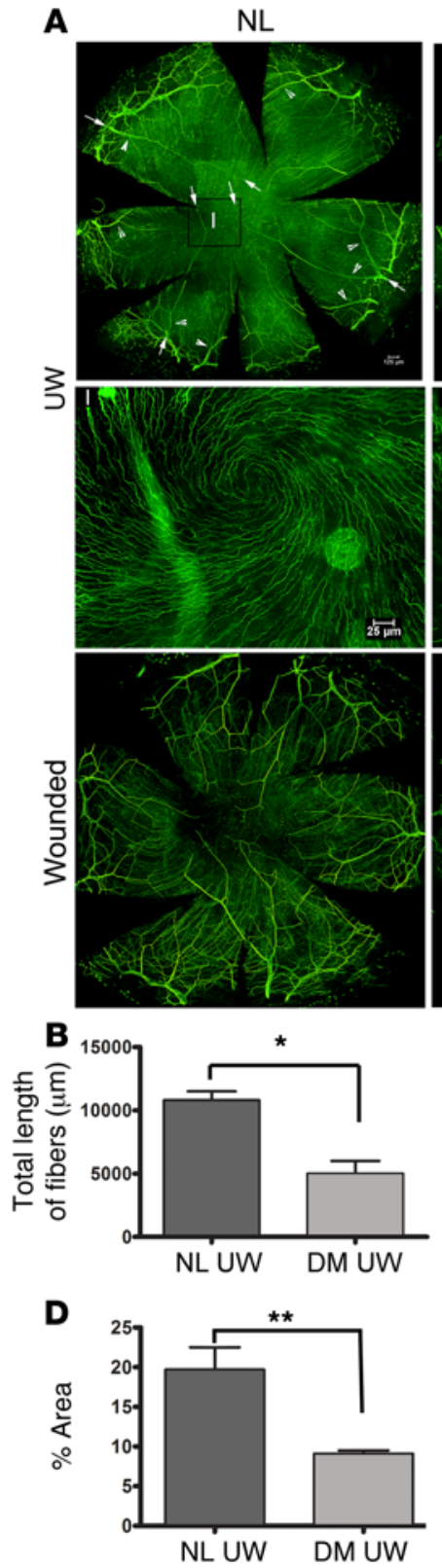

DM
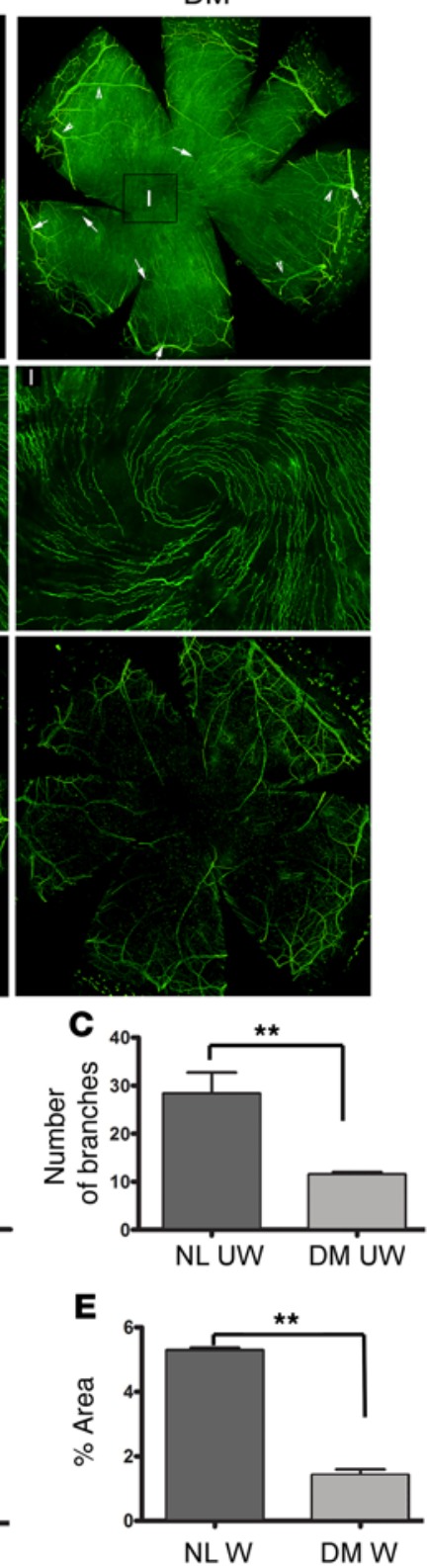

edge of the NL wounded corneas compared with that observed in DM wounded corneas. In the NL healing corneas, we detected 1 DC in front of each growing nerve fiber/ending (NL2, Figure 4B), whereas the growing nerve fibers of the DM healing cornea (DM2, arrows, Figure 4B) were fewer in number and lacked nearby DCs.

We also used flow cytometry to assess the decline in the number of DCs and MHC class II-positive cells in healing corneas (Figure 4C). In UW corneas, NL mice had $3.08 \% \pm 0.31 \%$ and DM mice had $1.62 \% \pm 0.23 \%$ CD11c- and MHC class II-positive cells out of the total live cell population; in healing corneas, NL mice had $3.08 \% \pm 0.31 \%$ and DM mice had $1.62 \% \pm 0.23 \%$ CD11c- and MHC class II-positive cells out of the total live cell population. In both UW and healing corneas, NL mice had significantly higher numbers of DCs than did DM mice (Figure 4D).

DCs in UW and healing corneas express CNTF. Loss of neurotrophic support has been implicated in DPN $(39,40)$. Our ini-

Figure 1. Quantitation of sensory nerves in UW and healing NL and DM mouse corneas. A 2-mm epithelial debridement wound was created and allowed to heal for 18 hours in NL corneas and for 24 hours in DM corneas, resulting in similarly sized remaining wounds. UW and healing corneas were stained with $\beta$-tubulin III Ab for sensory nerve labeling. The WMCM images were acquired with ROI scanning of a defined area and assembled automatically to show whole corneas from B6 mice (A). To quantify the innervation in UW corneas, the major nerve fibers, defined as those that extended to at least one-third of the corneal diameter, starting from the limbus, with or without branches, were marked (arrows); the length was measured; and the branches were manually counted for each marked fiber and presented as the total length (B) and number of branches (C) per cornea. Central areas with a swirling pattern of nerve endings (framed area "I" in $\mathbf{A}$, top panel) are shown at higher magnification (A, middle panel), and innervation was calculated with Image) as the percentage of area positive for $\beta$-tubulin III staining (D). Innervation in healing corneas was also calculated as the percentage of areas positive for $\beta$-tubulin III staining (E). Results are representative of 2 independent experiments ( $n=3$ each). ${ }^{* *} P<0.01$ and ${ }^{*} P<0.05$, by 2 -tailed, unpaired Student's $t$ test. Scale bars: $125 \mu \mathrm{m}$ (A, top and bottom panels) and $25 \mu \mathrm{m}$ (A, middle panel). W, wounded.

tial screening using regular reverse transcriptase PCR (RT-PCR) revealed that, while brain-derived neurotrophic factor $(B d n f)$ and neurotrophin-3 (Nt3) were constitutively expressed, the band intensities of nerve growth factor $(\mathrm{Ngf})$ and $\mathrm{Cntf}$ were higher in NL, but not DM, corneas (Supplemental Figure 3). To assess the quantitative changes in NTF expression, we first performed qPCR to screen for known NTFs (Figure 5A). We found that $N g f$ and Cntf had a significantly elevated expression levels in response to wounding, and this upregulation was attenuated in the DM corneas. The Cntf expression pattern was similar to that of Cd11c mRNA expression. While NGF has been shown to be expressed primarily in healing epithelial cells (41) and by $C D 11 \mathrm{~b}^{+} \mathrm{GR} 1^{+}$myeloid cells (42), the cells expressing CNTF in UW or wounded corneas were unknown. Hence, we focused on CNTF.

DCs in UW corneas of both NL and DM mice were CNTF positive, with faint staining (Figure $5 \mathrm{~B})$. In healing NL corneas, we detected abundant numbers of CD11c-positive infiltrates; all CD11c-positive cells were CNTF positive, while a few CNTFpositive cells were CD11c negative (Figure 5C). In DM corneas, the densities of both CD11c- and CNTF-positive cells were much lower, and the majority of CD11c-positive cells, with a few exceptions (enlarged images ["I"], Figure 5C), were CNTF positive (Figure 5C). Cell counting revealed significant decreases in the numbers of CD11c- and CNTF-positive cells in DM corneas compared with those in NL corneas (Figure 5D).

DCs mediate corneal reinnervation in a CNTF-dependent manner. Having shown that DCs are the major source of CNTF and that diabetes suppresses DC populations in UW and healing corneas, we next investigated the role of CNTF in corneal epithelial wound healing and reinnervation using CNTF-neutralizing Abs in NL corneas (Figure 6). Neutralizing CNTF showed no effects on epithelial wound closure (Figure 6, A and C). Compared with the control, CNTF neutralization markedly inhibited nerve-ending regeneration, and a near-absence of nerve endings was observed in the treated corneas at $1 \mathrm{dpw}$ (Figure 6B). The lack of nerve fiber and ending regeneration in the presence of CNTF-neutralizing $\mathrm{Ab}$ could be observed out to 7 days (Supplemental Figure 4). The extent of innervation, mostly the formation of sensory nerve end- 
A
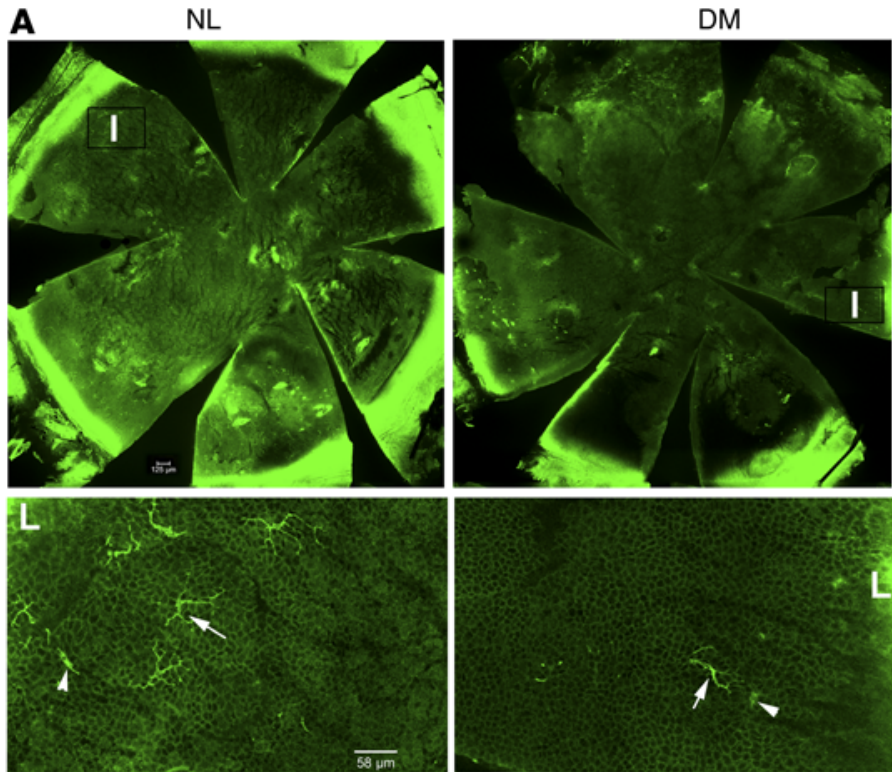

B
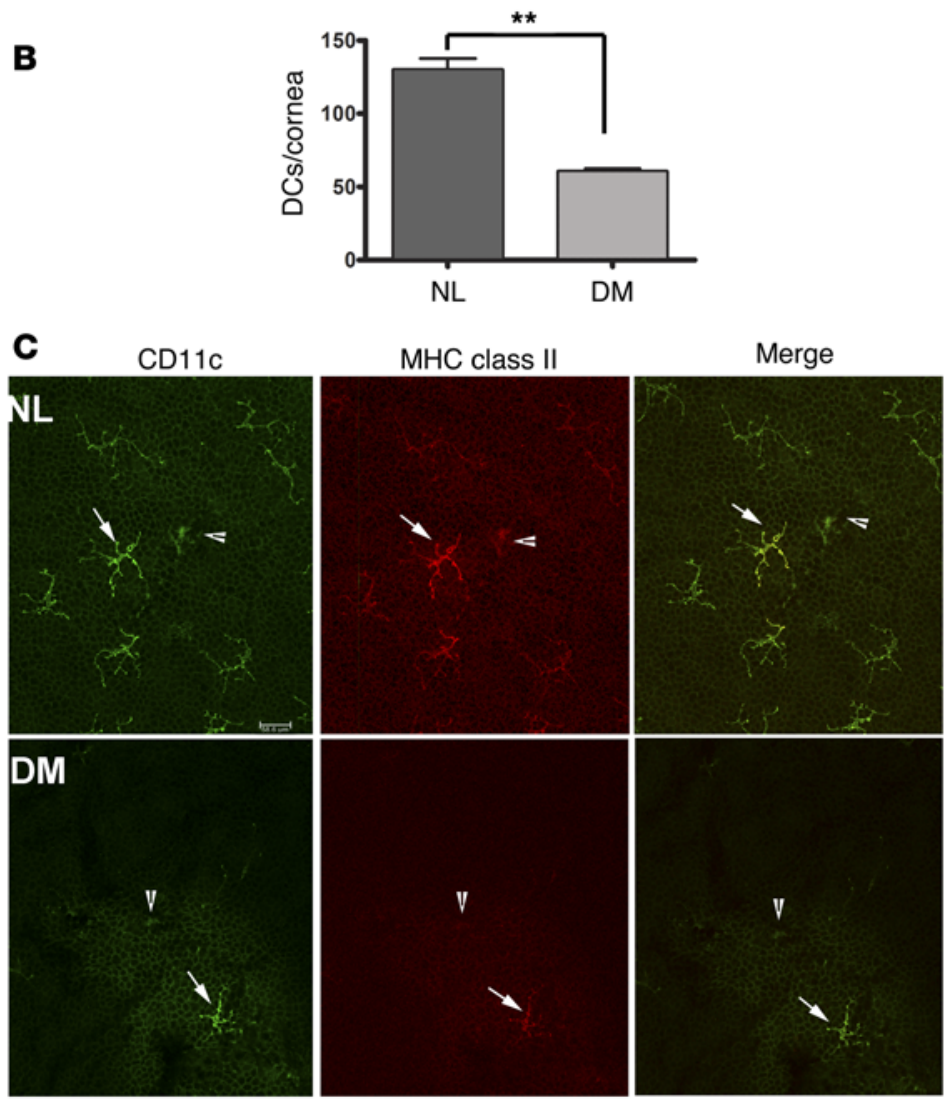

Figure 2. Quantitation of DCs in UW NL and DM mouse corneas. UW corneas were stained for CD11c and/or costained for CD11c and MHC class II and visualized with WMCM. (A) Whole-cornea images were assembled from WMCM images acquired with ROI scanning of a defined area as described in Figure 1. Two morphologically distinctive CD11c-positive cells were detected: dendriform (arrows) and rounded-shaped (arrowheads) DCs, which are better illustrated in the higher-magnification bottom panel images of the framed "I" areas in the top panel images. Scale bars: $125 \mu \mathrm{m}$ (top panel) and $58 \mu \mathrm{m}$ (bottom panel). L, limbus. (B) CD11c-positive cell numbers in UW corneas were counted manually. Results are presented as the average number of CD11c-positive cells (particles) per cornea. Three independent experiments were performed. ${ }^{*} P<0.01$, by 2 -tailed, unpaired Student's $t$ test. (C) Costaining for CD11c and MHC class II Abs. Two morphologically distinctive CD11c-positive cells, dendriform (arrows) and rounded-shaped DCs (arrowheads), were observed, and all CD11c-positive cells were also MHC class II positive in UW $\mathrm{NL}$ and DM corneas. Scale bar: $58 \mu \mathrm{m}$.

CNTF had minimal effects on the number of sensory neurons regenerated in the NL corneas (Figure 7B) and yet was likely to increase the numbers of branches near the limbal region. While large fibers with only a few small nerve endings were seen in the DM corneas (Figure 7C), CNTF treatment resulted in the formation of a fiber network with a higher density of nerve endings; the network, however, did not extend as far as that of the NL corneas (Figure 7D). We calculated the percentage of the selected area covered by tubulin staining and found that CNTF treatment significantly increased sensory nerve reinnervation in the DM corneas (Figure 7E).

CNTF/CNTFRa signaling is critical for sensory nerve regeneration in wounded corneas. The CNTFR $\alpha$ subunit provides the receptor specificity for CNTF (43). To assess the role of CNTFR $\alpha$ in sensory nerve homeostasis in UW corneas and in regeneration in wounded corneas, we either blocked CNTFR $\alpha$ activity in the NL corneas or applied soluble CNTFR $\alpha$ locally to the DM corneas (Figure 8). For NL corneas, CNTFR $\alpha$ activity was first blocked by subconjunctival injections of neutralizing $\mathrm{Ab}$ every other day, with the first injection on day 0 . These injections consisted of neutralizing $\mathrm{Ab}$ in the left eye or IgG as a control in the right eye. On day 9 (24 hours after the fifth injection), WMCM revealed that CNTFR $\alpha$ neutralization significantly reduced the density of sensory nerves, mostly in the sub-basal plexus of the corneas (Figure 8A). In wounded corneas, CNTFR $\alpha$-neutralizing Ab was injected 4 hours before epithelial debridement,

ings branched from fibers, in NL corneas was significantly higher than that in DM corneas, and CNTF neutralization had little effect on epithelial innervation in the DM corneas (Figure 6D).

We next performed subconjunctival injection of recombinant CNTF to determine whether replenishing CNTF accelerates sensory nerve regeneration in the healing corneas of DM mice (Figure 7). Four hours before 2-mm epithelial debridement, $50 \mathrm{ng}$ recombinant CNTF was subconjectivally injected, and nerve regeneration was examined $1 \mathrm{dpw}$. A network of nerve fibers with numerous nerve endings was growing from the limbal region (Figure 7A); and the regeneration of sensory nerves was significantly impaired by CNTFR $\alpha$ neutralization at $2 \mathrm{dpw}$, as the tubulin-stained area was reduced compared with that seen in the IgG-injected corneas. We obtained similar results using the complementary approach of siRNA technology to downregulate CNTFR $\alpha$ (Figure 8B). Longterm CNTFR $\alpha$ siRNA treatment decreased sensory nerve density and siRNA levels when injected 24 and 4 hours before wounding, impairing nerve regeneration in the NL corneas. Hence, CNTF/ CNTFR signaling plays an important role in sensory nerve maintenance and regeneration. 
A
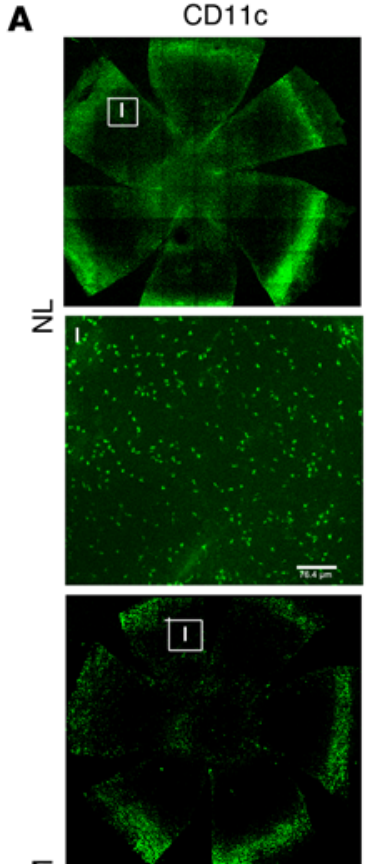

¿

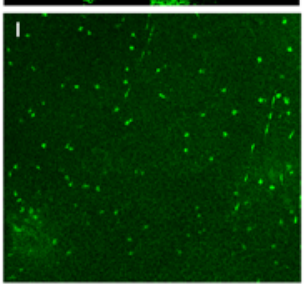

B

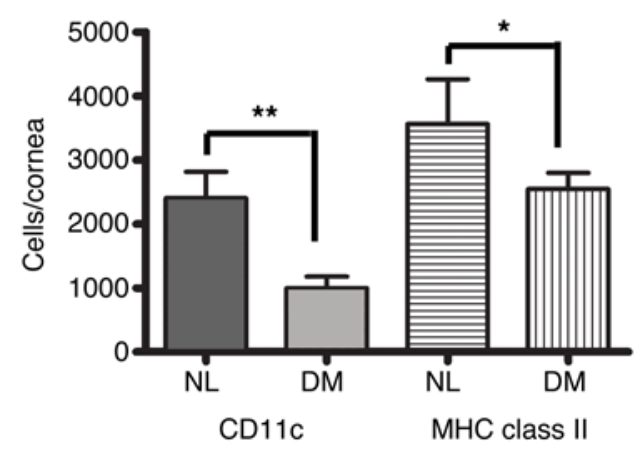

Soluble forms of CNTFR $\alpha$ have been detected in serum and cerebrospinal fluid (44) and shown to enhance CNTF-mediated protection against neuronal death and neurite outgrowth (45). Soluble CNTFR $\alpha$ can reconstitute a high-affinity-type CNTFR complex (46). Hence, we postulated that the addition of recombinant (soluble) CNTFR $\alpha$ may increase CNTF signaling in DM corneas with reduced CNTF levels. To test this hypothesis, we administered soluble CNTFR $\alpha$ to UW corneas for 10 days or prior to epithelial wounding. While the presence of exogenous CNTFR $\alpha$ had no apparent effect on the density of nerves in whole, UW corneas of DM mice, its presence in the healing corneas resulted in a significant increase in sensory nerve regeneration, with more nerve endings branching out from small nerve fibers and extending further into the center of the wounds in the DM corneas (Figure 8C).

CD11c-positive cells are responsible for CNTF production in the cornea. To determine whether CD11c-positive cells are responsible
Figure 3. Quantitation of DCs in healing NL and DM mouse corneas. Corneas were wounded and allowed to heal for 22 hours Wounded corneas were stained with CD11c and MHC class II Abs, and whole-cornea images were generated as described in Figure 2 (A). A selected area (framed "I" area) was enlarged to show CD11c-positive and MHC class II-positive cells and their colocalization (bottom panel). Note: While all CD11c-positive cells were also MHC class II positive (yellow staining), some MHC class II-positive cells were CD11c negative. Scale bars: $455 \mu \mathrm{m}$ and $76.4 \mu \mathrm{m}$ (highermagnification panels). (B) All CD11c- and MHC class II-positive cells, excluding those in the densely packed area representing the limbal region, were quantitated using the particle-counting function in Image J. Results are presented as the average number of CD11c- or MHC class II-positive cells (particles) per cornea $(n=3) .{ }^{*} P<0.01$ and ${ }^{* *} P<0.01$, by 2 -tailed, unpaired Student's $t$ test. Two independent experiments were performed.

for wound-induced production of CNTF in the cornea, we used $\mathrm{Tg}$ mice that express a $C D 11 c$ promoter-driven diphtheria toxin (DT) receptor (B6-DTR), with WT B6 mice used as controls (47). Our previously published data showed that subconjunctival injection of DT allowed the local depletion of corneal DCs, without affecting splenic DCs (33) (see also supplemental Supplemental Figure 5). DT had minimal effects on sensory nerve regeneration in WT B6 mouse corneas, whereas DTR mice without DT injection behaved similarly to WT B6 mice (Figure 9). While DC depletion resulted in impaired nerve regeneration, the presence of CNTF counteracted the adverse effect of DC depletion and resulted in a robust sensory nerve-ending regeneration, although the endings appeared to be shorter than those in the corneas of control Tg mice without DC depletion (Figure 9A). Twoway ANOVA revealed that exogenous CNTF restored the impaired sensory nerve-ending regeneration to a level similar to that seen in the controls (Figure 9B).

\section{Discussion}

We previously demonstrated that diabetes causes delayed epithelial wound healing and impairs sensory innervation in the corneas $(8,9)$. However, the cellular and molecular mechanisms underlying this were not known. In the present study, we showed that there are significant losses of sensory nerve fibers and endings and marked decreases in DC populations in DM mouse corneas. In NL healing corneas, we detected a large number of infiltrated DCs that were distributed among newly generated nerve endings. In DM healing corneas, the numbers of DCs and regenerating nerves were greatly decreased. Furthermore, the newly grown nerves were fewer in number and abnormal in morphology, with a reduced DC population between these nerves in the DM corneas. We showed that DCs are the major source of CNTF. Moreover, whereas there were no effects on epithelial wound closure, neutralizing CNTF severely impaired the regeneration of nerve endings during the healing process. Exogenous CNTF, on the other hand, had a minimal effect on NL corneal nerve regeneration, but greatly increased nerve-ending branching from nerve fibers in wounded DM corneas. Finally, we showed that CNTFR $\alpha$ is required for maintenance of the sub-basal nerve plexus in UW corneas and for nerve regeneration in the healing corneas 
A

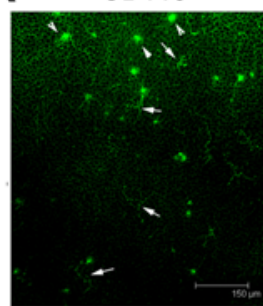

B

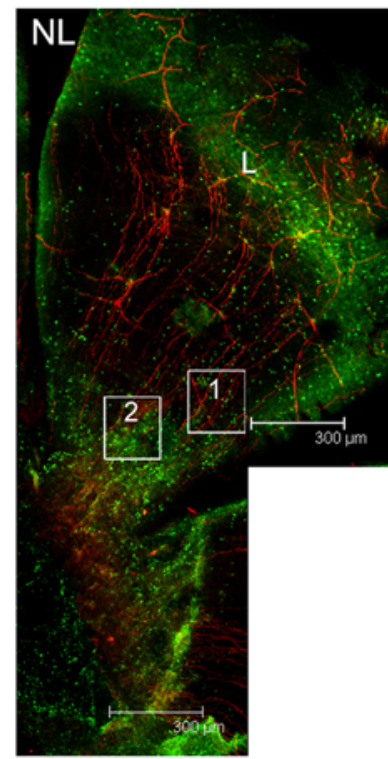

$\beta$-Tubulin III

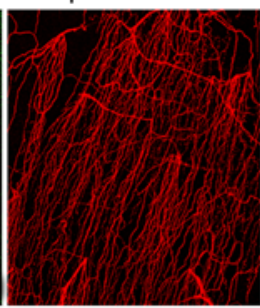

Merged

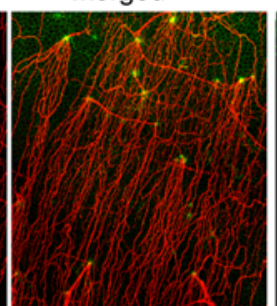

CD11c

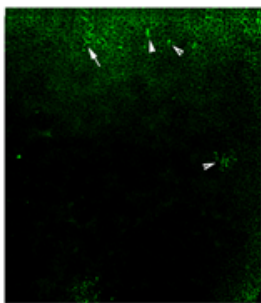

$\beta$-Tubulin III

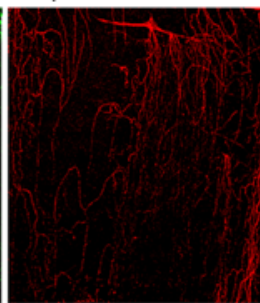

Merged

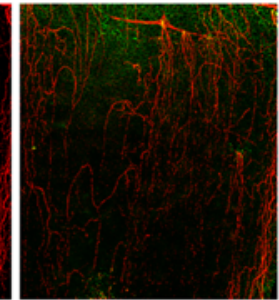

NL
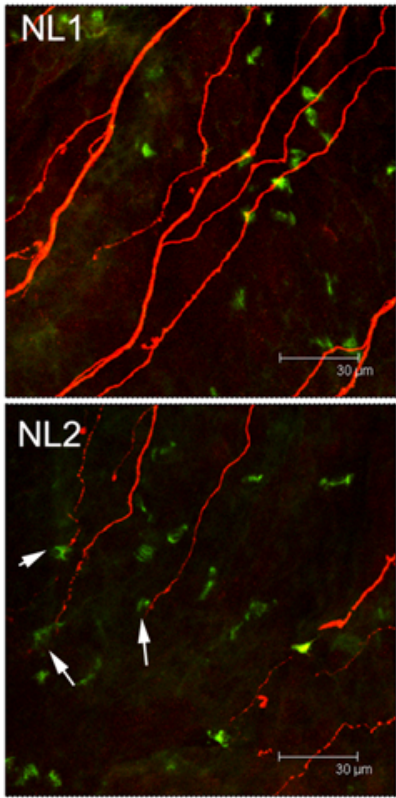

DM
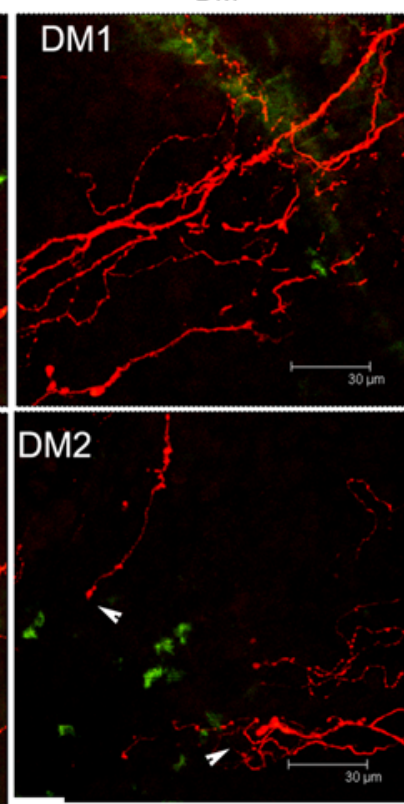

DM

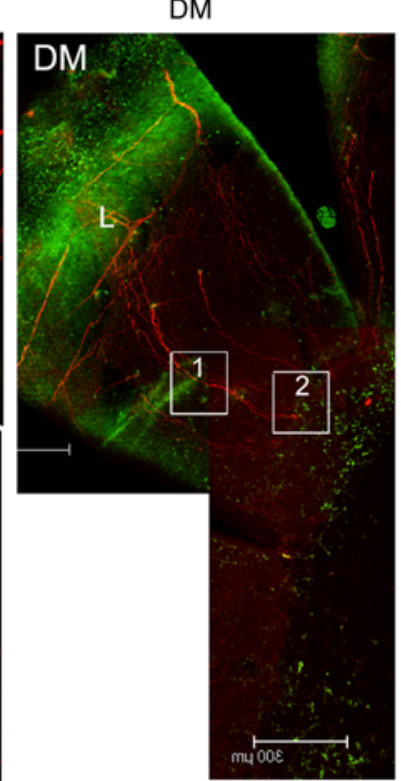

\section{$\mathbf{C}$}

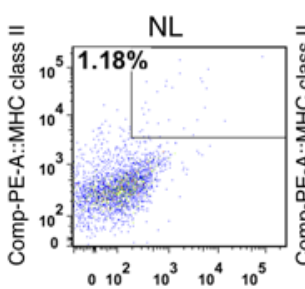

Comp-PE-Cy7-A::CD11c

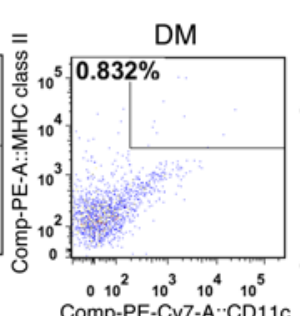

Comp-PE-Cy7-A::CD11c

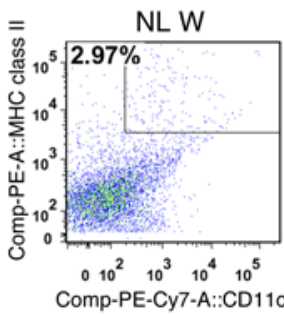

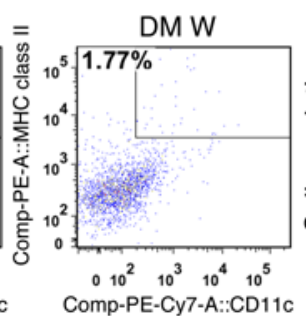

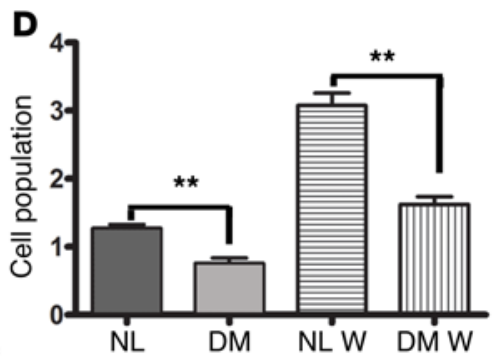

Figure 4. DCs and sensory nerve fibers and endings in healing NL and DM mouse corneas. (A) Whole-mount, UW corneas were double stained with $\beta$-tubulin III and CD11C Abs. Micrographs were taken near the limbal region. Arrows indicate dendriform DCs; arrowheads indicate round-shaped DCs. Scale bar: $150 \mu \mathrm{m}$. (B) Corneas were wounded as described in Figure 1. The whole cornea from the limbus $(\mathrm{L})$ to the leading edge was photographed with $a \times 10$ lens (side panels). Higher-magnification images (original magnification, $\times 40$ ) at the middle (white-framed area 1 ) and leading edge (whiteframed area 2) of healing migratory sheets are shown in the middle panels. Arrows indicate colocalization of nerve endings with a DC in NL1 cornea; arrowheads indicate free nerve endings in DM cornea. Results are representative of 2 independent experiments $(n=3)$. Scale bars: $300 \mu \mathrm{m}$ (side panels) and $30 \mu \mathrm{m}$ (middle panels). (C) Flow cytometric analyses of CD11c-positive cells in UW and wounded (W) NL and DM corneas. Five corneas were pooled for each sample. Percentages of MHC class II- and CD11c-positive cells are shown in the flow cytometric plots. (D) Three independent experiments were performed, and after normalization with NL UW corneas, the percentage of CD11C- and MHC class II-positive cells in all living cells was analyzed with a 2-tailed, unpaired Student's $t$ test $(n=3) .{ }^{*} P<0.01$. Comp, compensation beads.

of NL mice. More important, exogenously added soluble CNTFR $\alpha$ partially restored the impaired nerve-ending regeneration seen in DM mouse corneas. Finally, local depletion of DCs impaired sensory nerve-ending regeneration, and this impairment was quantitatively overcome by exogenous CNTF, suggesting that CD11cexpressing cells were responsible for CNTF production in the B6 mouse cornea. Taken together, our study reveals a critical role for
DC-nerve interactions in corneal innervation and nerve regeneration and that a decrease in DC-produced CNTF is responsible, at least in part, for the defects in sensory nerve regeneration during epithelial wound healing in DM corneas.

Our study shows that DCs influence sensory innervation and regeneration and demonstrate, for the first time to our knowledge, that diabetes disrupts DC-nerve communication, resulting in 


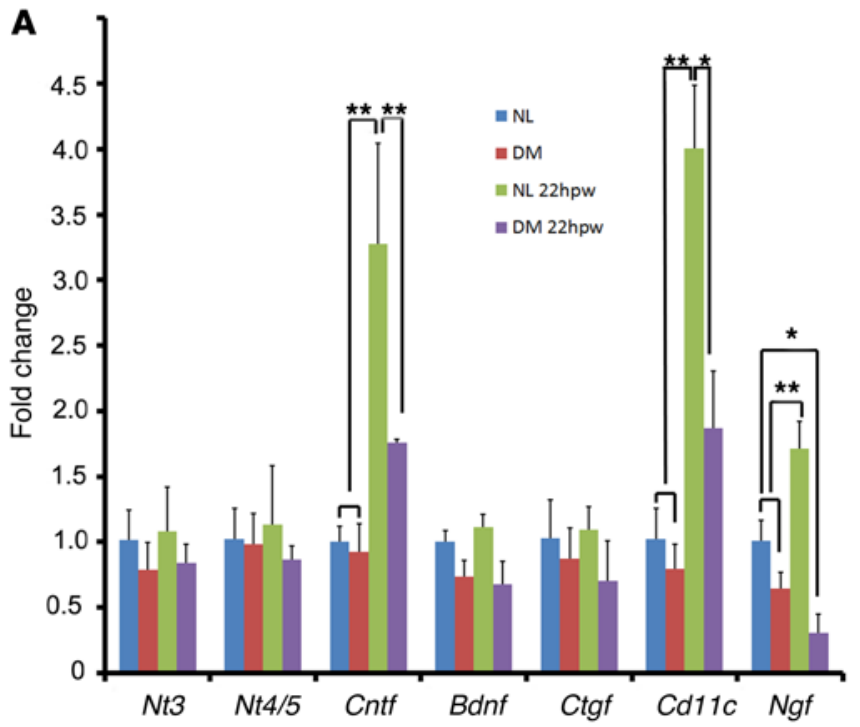

C
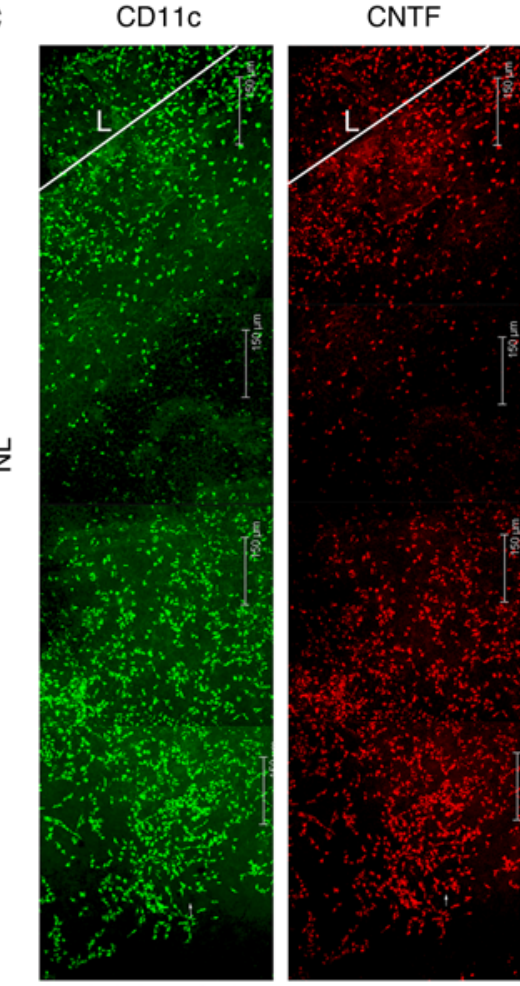

Merged
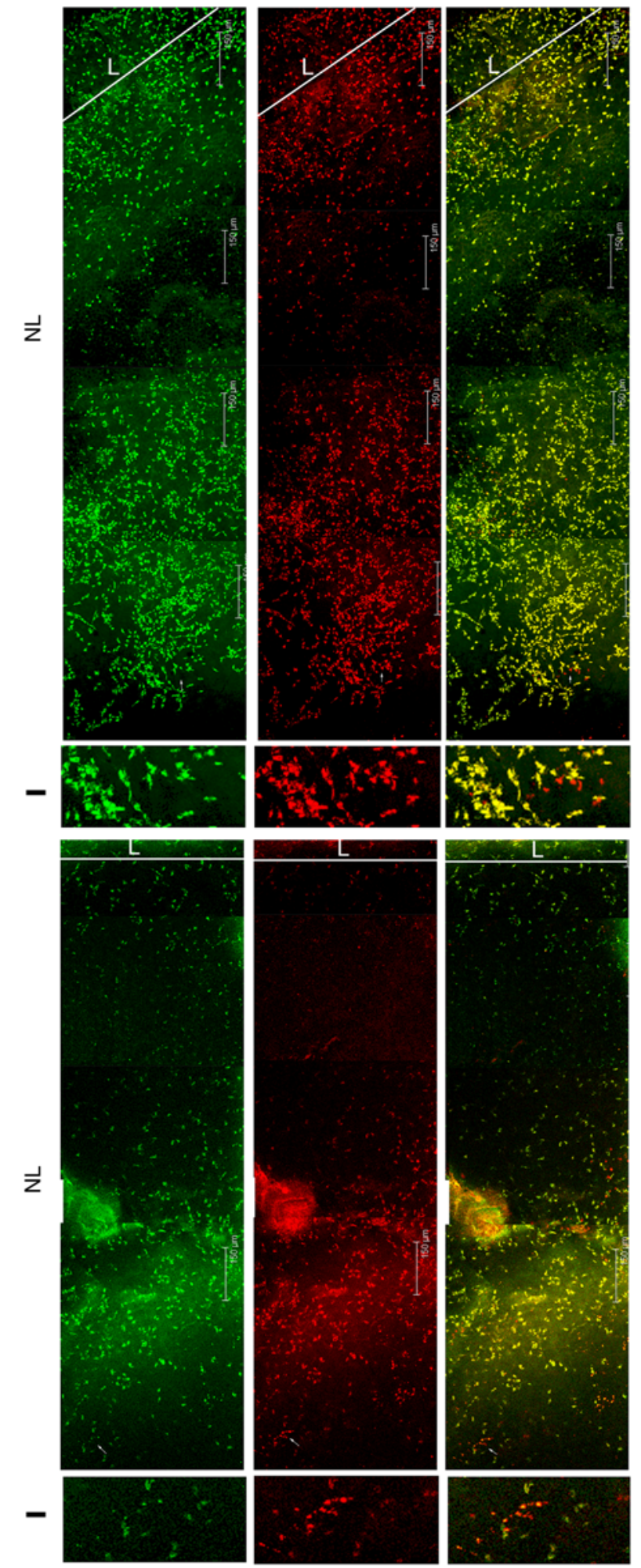

D

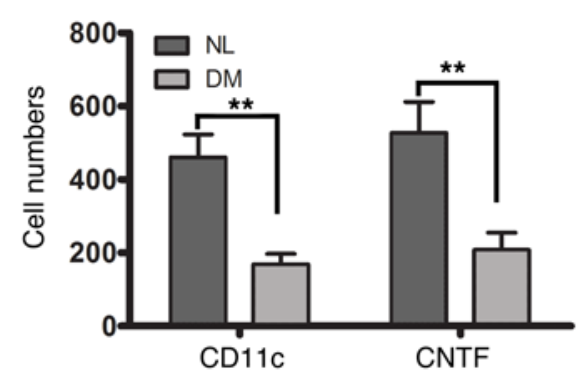

Figure 5. CNTF expression and colocalization with CD11c-positive cells in NL and DM corneas with or without epithelial debridement. Both NL and DM corneas were wounded as described in Figure 1. (A) UW and healing corneas (22 hpw) were collected and processed for RT-PCR screening of neurotrophins. Results were first normalized with $\beta$-actin levels and then compared with NL levels (value 1), presented as the fold change $(n=5)$. ${ }^{*} P<0.01$ and ${ }^{*} P<0.05$, by 2-way ANOVA with Bonferroni's post test. (B) WMCM showing colocalization of CD11c and CNTF in UW normal and DM corneas. Note that, although faint, CNTF red staining was also visible where CD11c-positive cells were located. Scale bar: $75 \mu \mathrm{m}$. (C) WMCM showing CD11c and CNTF colocalization in healing corneas of NL and DM mice. Enlarged bottom panel images ("I") show CD11c-negative and CNTF-positive cells. L, limbal region. Scale bars: $150 \mu \mathrm{m}$; original magnification, $\times 3.8$ (enlarged "I" images). (D) Both CD11C- and CNTF-positive cells in the corneas were quantitated using the particle-counting function in Image). Results are presented as the average number of CD11c- or CNTF-positive cells (particles) in the selected area $(n=3)$. ${ }^{* *} P<0.01$, by 2-tailed, unpaired Student's $t$ test. Two independent experiments were performed. 
$\mathrm{NL}$
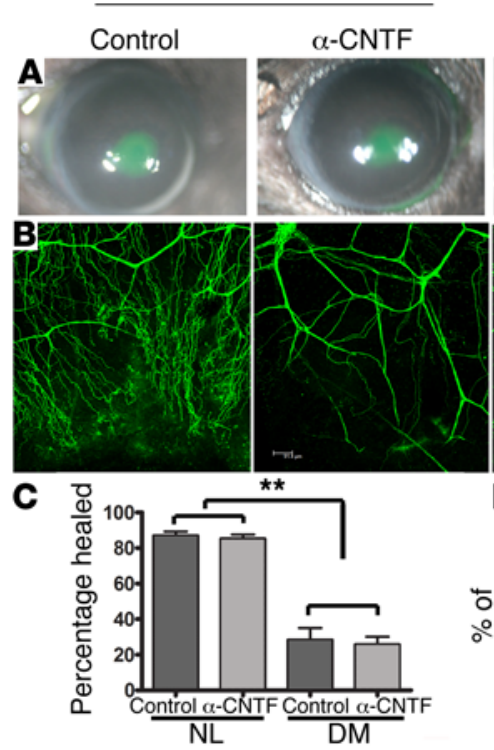

DM
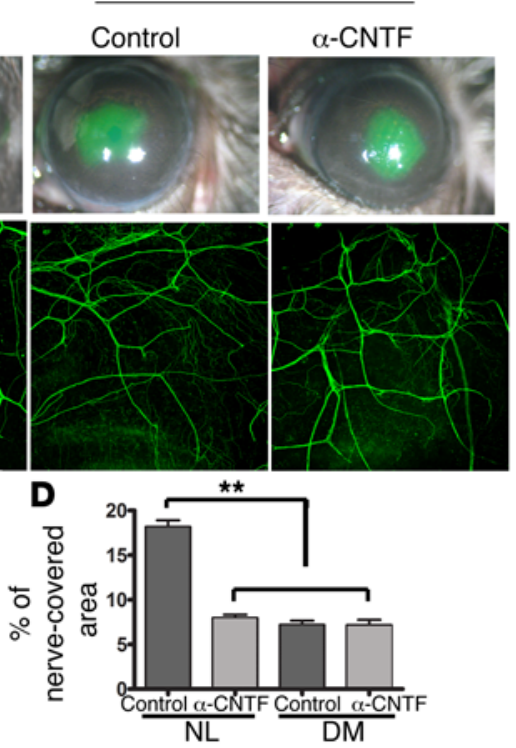

Figure 6. Effects of CNTF-neutralizing Ab on sensory nerve reinnervation in NL and DM mouse corneas. (A) NL and DM corneas were subconjunctivally injected with CNTFneutralizing $A b$ or IgC as the control 4 hours before epithelial debridement. A 2-mm wound was made at the center of the cornea and allowed to heal for 22 hours. The remaining fluorescence-stained wounds were photographed. Note the similar sizes of the wounds with or without CNTF-neutralizing Ab treatment. (B) Regeneration of sensory nerve endings was assessed by WMCM at $1 \mathrm{dpw}$ with $\beta$-tubulin III staining, and the micrographs were taken near the limbal region. Note the lack of sensory nerve endings in the CNTF-neutralizing Ab-treated corneas. Scale bar: $91.4 \mu \mathrm{m}$. (C) Wound-healing rates presented as the percentage of healed cornea 22 hours after epithelial debridement $(n=5)$. ${ }^{* *} P<0.01$, by 2 -way ANOVA with Bonferroni's post test. (D) Corneal innervation was quantified as the percentage of threshold area positive for $\beta$-tubulin III staining in the representative confocal images shown in B. Results are representative of 2 independent experiments ( $n=5$ each). ${ }^{* *} P<0.01$, by 2 -way ANOVA with Bonferroni's post test. impaired peripheral sensory nerve regeneration. In the cornea, a decrease in the density of sensory nerves occurs during the early stages of diabetes $(8,9,16-18)$. On the other hand, the effects of diabetes on DCs are less clear. We quantitated the total number of DCs in a mouse corneas and found decreased numbers of CD11c-positive DC populations in UW and healing DM corneas compared with those in NL corneas. In diabetic UW corneas, we observed relatively fewer CD11c-positive cells, particularly those associated with epithelium-innervating nerves near the limbal region, compared with the numbers detected in NL corneas. This decrease is consistent with a decrease in the density of nerve fibers and endings. Our results differ from those reported recently by Leppin et al., who showed that there was a substantial increase in mature DCs colocalizing with nerve fibers in DM corneas (32). One explanation for this difference is that, while we only stained the cornea with CD11c, Leppin et al. used a mix of CD11c and MHC class II Abs, which may also stain macrophages and fibroblasts in inflamed corneas $(48,49)$. Indeed, we observed a marked decrease in the infiltration of CD11c-positive cells and proportionally more MHC class II-positive cells than CD11c-positive cells in DM corneas in response to wounding. In addition, our WMCM results were verified by flow cytometry and qPCR and RT-PCR. Moreover, our results are consistent with those of recently published studies showing that the cutaneous density of Langerhans cells was profoundly reduced in recently diagnosed type 2 diabetes (50) and that healing diabetic foot ulcers showed higher numbers of Langerhans cells than did nonhealing ones (51). We found that the abnormalities of sensory nerve fibers and endings in DM corneas were more apparent when they were wounded: the reinnervation process was markedly delayed compared with the time required for epithelial wound closure, and substantially fewer fibers were formed, with almost no epithelial nerve endings. In addition, the newly formed fibers were morphologically distorted. These abnormalities were associated with the substantial decrease in DC populations in healing DM corneas. Our recently published data showed that diabetes affects the wound-induced expression of CXCL10 in the cornea (52). Since CXCL10 was linked to DC mobilization in the lung (53), the suppressed expression of CXCL10 may represent an underlying mechanism for the diabetes-driven disruption of DC infiltration. These results are in line with our conclusion that diabetes disrupts DC-nerve communication, resulting in DPN and impairment of sensory nerve regeneration in the cornea, and suggest that DC-based cell therapies have potential for the treatment of DPN in diabetic patients.

Our study is also the first to our knowledge to demonstrate that DCs are the major source of CNTF, a NTF that is necessary for peripheral nerve integrity and regeneration $(21,54)$. CNTF is a member of the NTF family (55), and its expression in DCs has not been reported. In the present study, we showed that CNTF expression is induced by wounding in normal corneas and is reduced in DM corneas. In the cornea, although CNTF has been shown to promote nerve fiber regeneration in incited mouse corneas (56) and is expressed in the epithelium of keratoconus corneas (57), wound-induced CNTF expression has not been reported (58, 59). In UW corneas, CD11c-positive cells are also CNTF positive, suggesting that through the production of CNTF, DCs may participate in maintaining the delicate structures of sensory nerves in the corneas. This was shown to be the case by neutralizing CNTF or its specific receptor CNTFR $\alpha$, which resulted in a decrease in the density of sensory nerve fibers and endings and a reduction in nerve-ending branching in UW NL corneas. By using 2 complementary approaches, we showed that CNTFR $\alpha$ is required for maintaining the sub-basal nerve plexus in UW corneas and for the branching of nerve endings in wounded corneas. These data indicate a critical role for CNTF/CNTFR $\alpha$ signaling in corneal innervation. More important, replenishing CNTF or CNTFR $\alpha$ partially restored reinnervation and the development of sensory nerve endings in DM corneas. Hence, we conclude that diabetes alters CNTF levels through the impairment of DC infiltration and that reduced DC populations and/or decreased CNTF levels in infiltrated DCs are responsible, at least in part, for DPN and for the impairment of neuron regeneration in wounded corneas. 

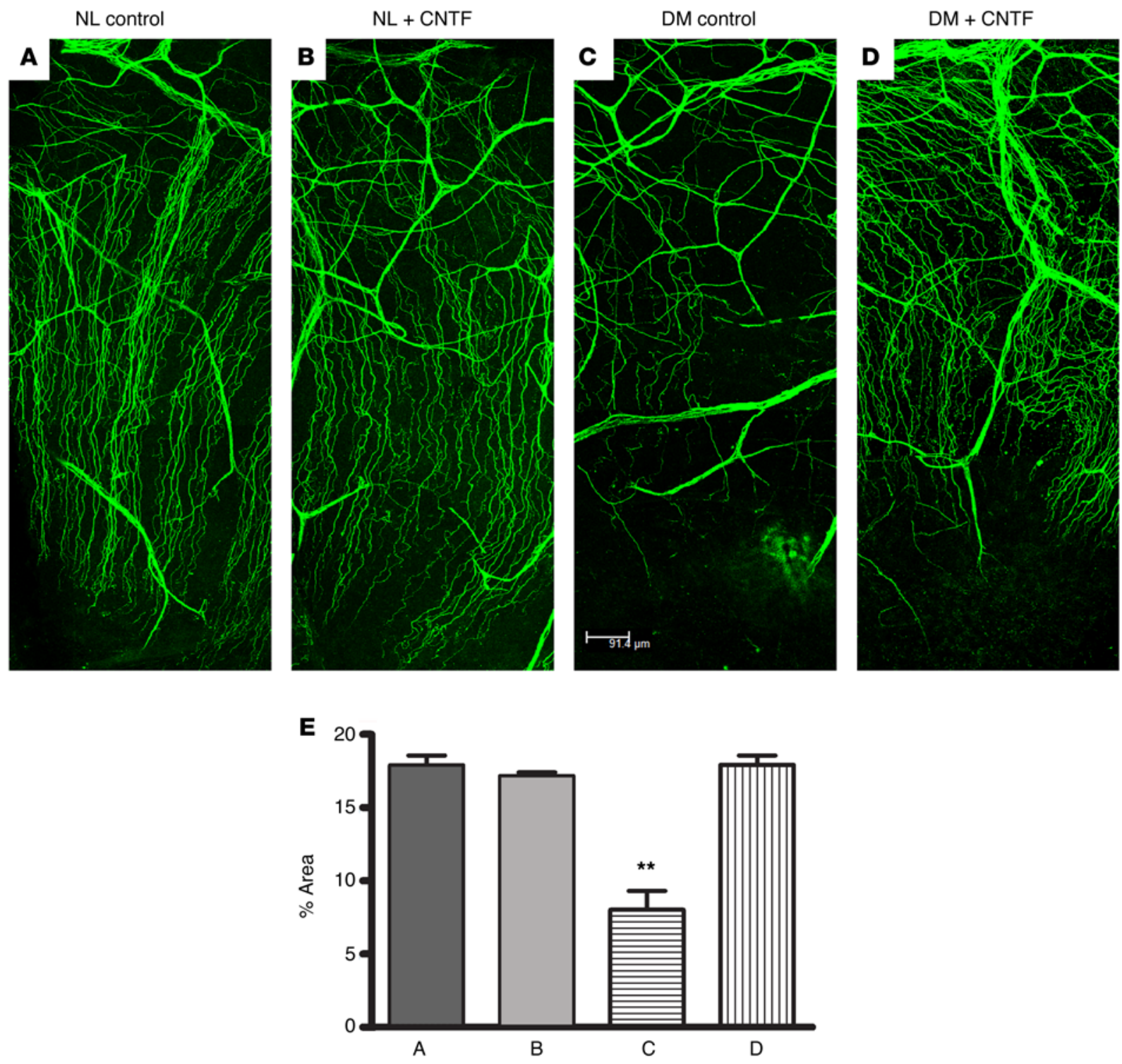

Figure 7. CNTF-enhanced sensory nerve regeneration in DM healing corneas. Both NL (A and $\mathbf{B}$ ) and DM (C and $\mathbf{D})$ corneas were subconjunctivally injected with $0.1 \%$ BSA as the control (A and C) or with recombinant CNTF (B and D) 4 hours before epithelial debridement. Corneas were stained with $\beta$-tubulin III 24 hpw and examined with WMCM. Micrographs were taken near the limbal region where large nerve fibers can be seen. (E) Corneal innervation was quantified as the percentage of threshold area positive for $\beta$-tubulin III signal in the representative confocal images shown in A-D. Results are representative of 2 independent experiments ( $n=5$ each). ${ }^{*} P<0.01$, DM control versus all other conditions, by 2-way ANOVA with Bonferroni's post test. Scale bar: $91.4 \mu \mathrm{m}$.

CNTF appeared to have no effects on corneal epithelial wound healing in NL or DM corneas. This result contradicts a recently published study showing that supplementing CNTF promotes diabetic corneal epithelial wound healing, accompanied by the regeneration of corneal nerve fibers, although no data on nerve regeneration were shown (60). A recent study showed that CD11b-positive cells, originating from $\mathrm{BM}$, secrete NGF and promote neuron regeneration in mouse corneas that underwent excimer laser annular keratectomy (42). It is possible that both elevated levels of NGF and CNTF are required for proper nerve regeneration in the cornea; the lack of either one could result in the aberrant axon sprouting we observed in DM and/or DC-depleted corneas (Figure 9).

Another important discovery of our study is that recombinant, soluble CNTFR $\alpha$ can restore sensory nerve integrity in UW
DM corneas and reverse impaired sensory nerve regeneration in wounded corneas. Human and mouse CNTF does not have the capacity to induce the heterodimerization of gp130 and leukemia inhibitory factor receptor $\beta$ (LIFR $\beta$ ) and requires the formation of a CNTF-CNTFR $\alpha$ dimer that recruits gp130 and LIFR $\beta$ to form the signaling complex (61). In addition to CNTFR $\alpha$, CNTF can use both the membrane-bound and the soluble human IL-6 receptor (IL-6R) as a substitute for its cognate $\alpha$ receptor and thus widen the target spectrum of CNTF binding (62). This capacity to signal through an IL-6R $\alpha$-gp130-LIFR $\beta$ tripartite receptor might contribute to the side effects observed in patients upon systemic administration of CNTF $(61,62)$. In addition to CNTF, cytokine-like factor 1 (CLF1) is also known to signal through CNTFR $\alpha$-gp130-LIFR $\beta$. CLF1, however, can activate only the tripartite CNTFR. Hence, exogenous, soluble 
A UW $2 \mathrm{dpw}$
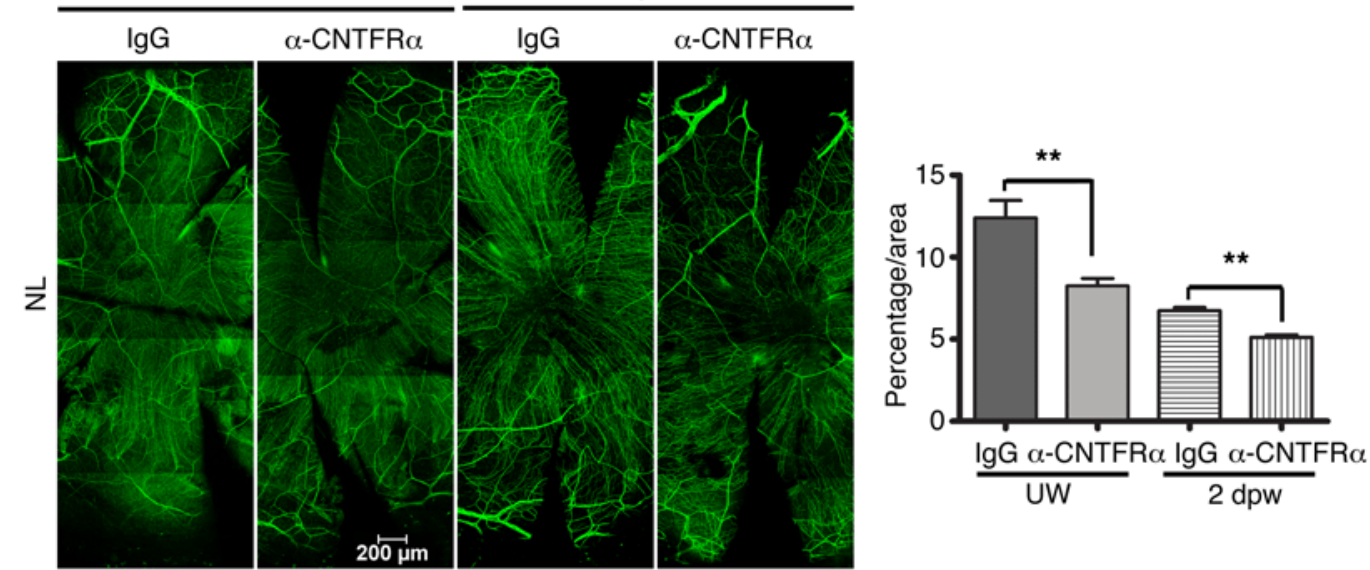

B Control

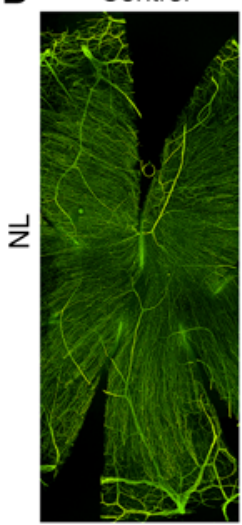

CNTFR $\alpha$

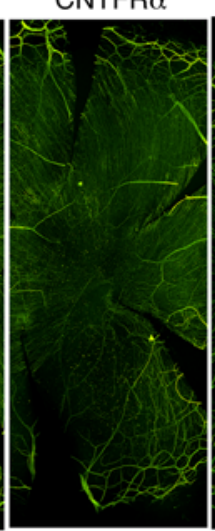

Control

CNTFR $\alpha$ (siRNA)
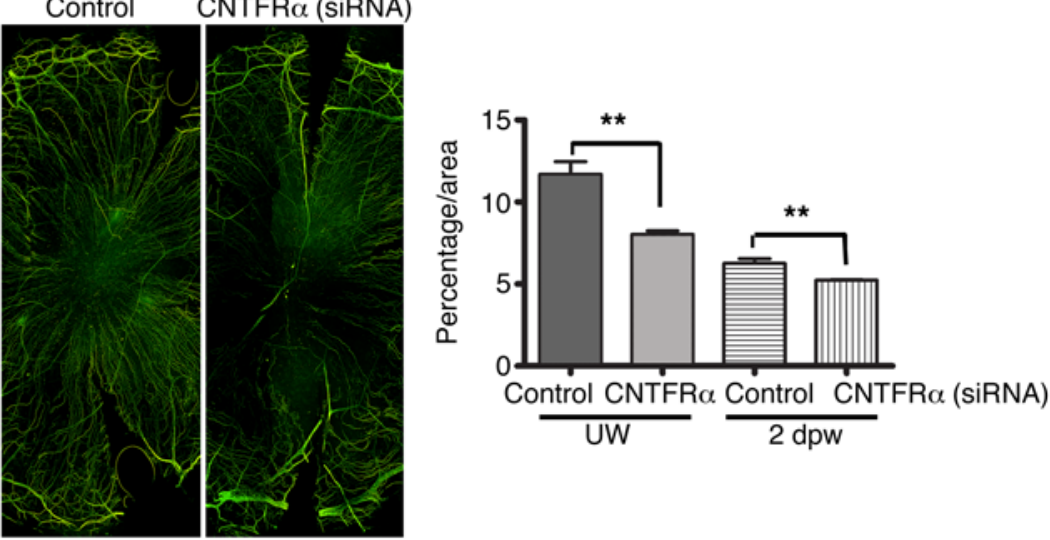

C

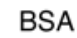

CNTFR $\alpha$

BSA

CNTFR $\alpha$ (soluble)
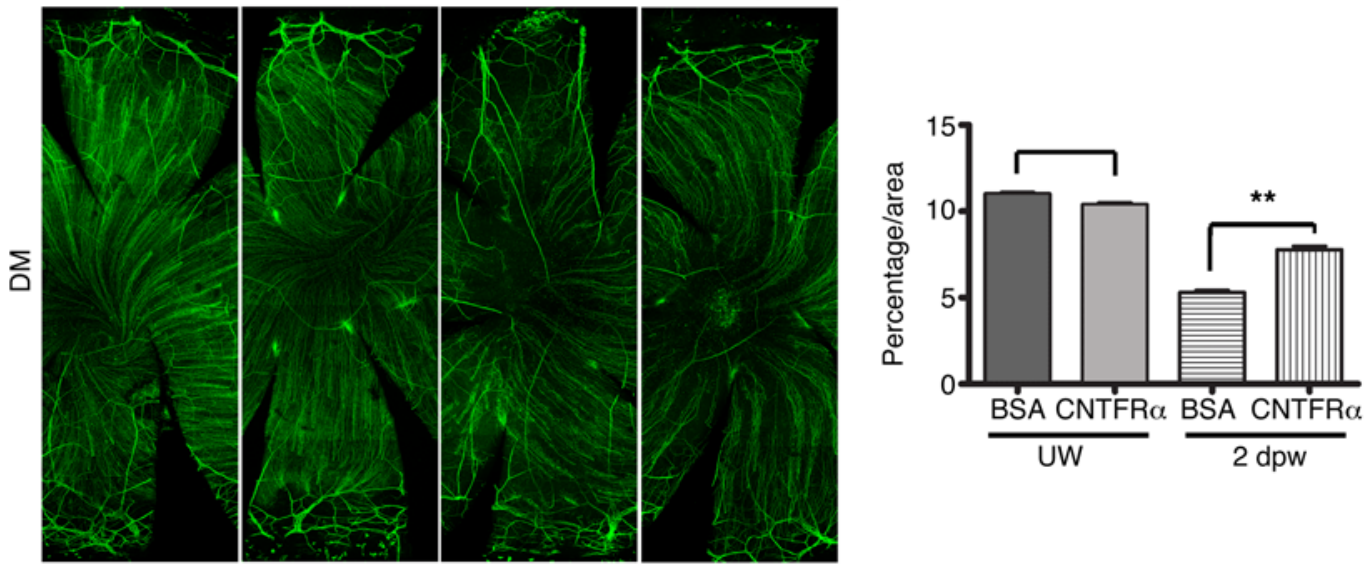

Figure 8. Effects of CNTFR $\alpha$ on sensory nerve reinnervation in NL and DM mouse corneas. In NL mice, CNTFR $\alpha$-neutralizing Abs or lgG as the control were subconjunctivally injected 5 times each every other day for the UW corneas or once 4 hours before epithelial debridement. (A) UW corneas on day 10 and wounded corneas $2 \mathrm{dpw}$ were collected, stained with $\beta$-tubulin III Ab for sensory nerve labeling, and examined with WMCM. Corneal innervation was quantified as the percentage of threshold area that stained positive for $\beta$-tubulin III in 3 corneal confocal images. Results are representative of 2 independent experiments ( $n=3$ each). ${ }^{*} P<0.01$, by 2-tailed, unpaired Student's t test. (B) In NL mice, CNTFR $\alpha$ siRNA or the control, nonspecific siRNA was subconjunctivally injected, as with the neutralizing Abs, into UW corneas, or injected twice 24 and 4 hours before epithelial debridement. Sensory nerve innervation and regeneration were examined and the results presented for the images in B. (C) In DM mice, UW corneas were subconjunctivally injected with recombinant CNTFR $\alpha$ every other day for 10 days or once 4 hours before epithelial debridement. Corneal innervation and nerve regeneration were examined and the results presented for for the images in C. All results are representative of 2 independent experiments $\left(n=3\right.$ each). ${ }^{* *} P<0.01$, by 2-tailed, unpaired Student's $t$ test. Scale bar: $200 \mu \mathrm{m}$. 
A

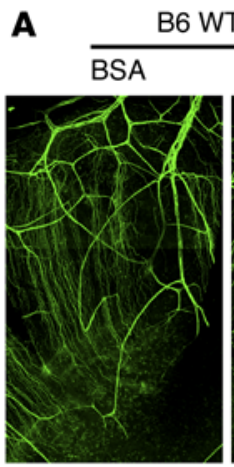

$6 \mathrm{WT}+\mathrm{DT}$

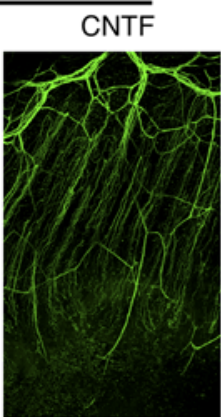

B
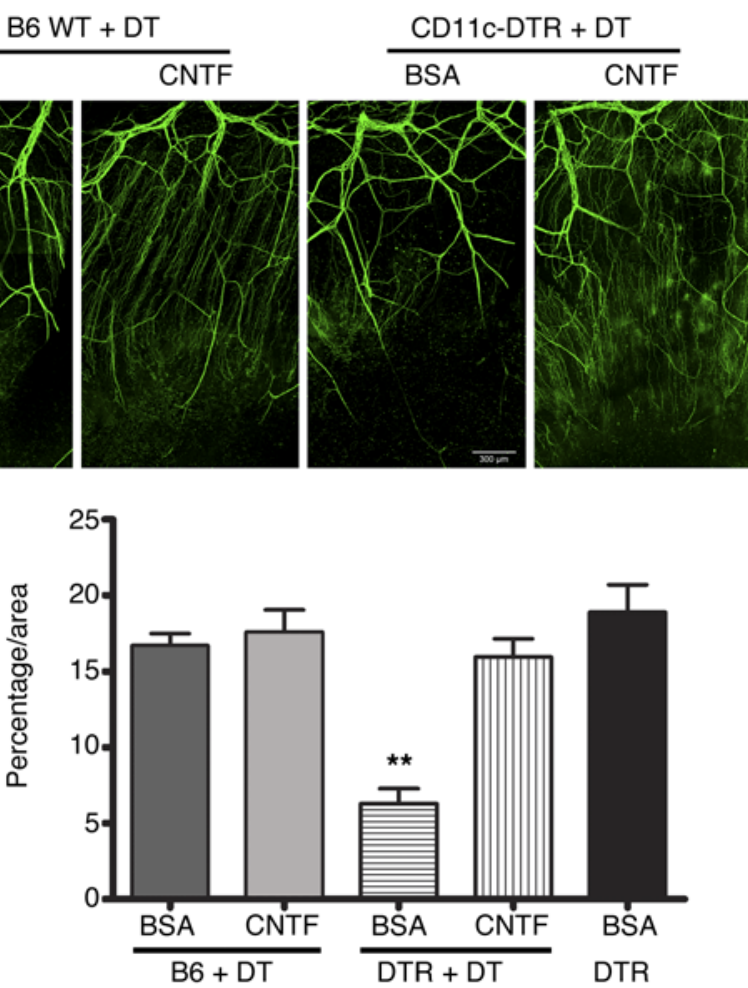

CD11c-DTR BSA

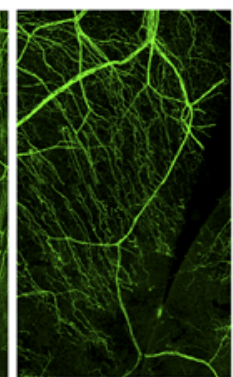

Figure 9. Effects of exogenous CNTF on sensory nerve regeneration in corneas with locally depleted CD11c-positive cells. (A) CD11c-positive cells were depleted from B6-CD11c-DTR, but not control B6, mice, by subconjunctival injection of DT 1 day before epithelial debridement. Four hours before epithelial debridement, DC-depleted and control (B6 WT mouse corneas injected with DT and B6-CD11c-DTR mice without DT injection) corneas were wounded and allowed to heal for 1 day. Corneas were stained for sensory nerve identification with -tubulin III Ab and examined using WMCM. Note that a lack of sensory nerve endings was apparent in CD11c-depleted corneas without exogenous CNTF. (B) Innervation was quantitated using ImageJ software, and the results are shown as the percentage of areas covered with CD11c staining in the images in $\mathbf{A}$. Results are presented as the mean \pm SD and are representative of 2 independent experiments $(n=$ 3 each). ${ }^{*} P<0.01$, CD11c-depleted corneas without exogenous CNTF versus all other conditions, by 2-way ANOVA with Bonferroni's post test.

CNTFR can define the receptor specificity for CNTF, control the agonist specificity of the CNTFR signaling complex, and increase CNTF signaling efficiency by forming more CNTF-CNTFR dimers, which in turn more effectively recruits gp130 and LIFR $\beta$ to form the tripartite CNTFR. These underlying mechanisms give CNTFR $\alpha$ the capacity to repair the impaired sensory nerve regeneration in DM corneas, where tissue levels of CNTF are low. Since soluble CNTFR $\alpha$ can limit or eradicate the undesired side effects of CNTF-IL-6R $\alpha$ or the CLF1 tripartite CNTFR, we propose that CNTFR $\alpha$ may be a valuable alternative to CNTF for the treatment of neurodegenerative diseases such as DPN (21), diabetic retinopathy (63), and age-related macular degeneration (64-66).

In summary, our data show that diabetes disrupts DC-nerve communication in UW corneas and during wound healing, resulting in DPN or DNK. These results suggest the potential of DC-based cell therapy, as autologous DCs can be readily prepared and manipulated to produce CNTF, and provide evidence for $\mathrm{CNTF}$ or its specific receptor $\mathrm{CNTFR} \alpha$ as a therapeutic reagent for the treatment of DPN.

\section{Methods}

Mice. WT C57BL6 (B6) mice (8 weeks of age; 20-24 g body weight) and CD11c-DTR (B6.FVB-Tg [Itgax-DTR/EGFP] 57Lan/J) breeding pairs were obtained from The Jackson Laboratory. B6-DTR mice carry a Tg encoding a simian DTR under the control of the murine Cd11c promoter, which makes cells expressing CD11c sensitive to DT. This allows the local depletion of DCs in the cornea (33).

B6 and B6-DTR mice were induced to develop type 1 DM according to a low-dose STZ (50 mg STZ/kg mouse) induction mouse protocol, without fasting prior to STZ injections. Glucose levels and body weight were monitored weekly. Animals with blood sugar levels higher than $350 \mathrm{mg} / \mathrm{dl}$ were considered diabetic and were used, with agematched animals as controls, 10 weeks after STZ injections $(8,23,67)$.

Immunostaining of whole-mount corneal tissue. To semiquantitatively assess the extent of corneal innervation and to quantify DCs and other cells expressing CNTF in the corneas, WMCM was used. Corneas excised at the indicated times (Figures 1-9) were fixed in 4\% paraformaldehyde (Electron Microscopy Sciences) and stored at $4^{\circ} \mathrm{C}$ until further processing. Whole-mount staining of DCs with anti-CD11c (clone HL3; BD Biosciences) and/or anti-MHC class II Abs (clone NIMR-4; eBioscience), anti- $\beta$-tubulin III Abs (clone TUJ1; Covance), and/or CNTFexpressing cells with anti-CNTF Abs (clone R-20; Santa Cruz Biotechnology Inc.) was performed as previously described $(9,33)$. Corneal whole mounts were examined using a Leica TCS SP2 confocal microscope or a Nikon ECLIPSE 90i microscope. Whole corneal WMCM images (Figures 1-3) were obtained using recorded images of regions of interest (ROI) scanning and automatically assembled from the acquired scanning images of a defined area in the TCS SP2 microscope. The images were presented without adjusting the background. To quantify corneal innervation, manual labeling and measurements were used in Image J software (NIH). For UW, whole corneas, fibers that extended from the limbus and past a distance measuring one-third of the corneal diameter were marked; their length was measured and the branches manually counted for each marked fiber. Innervation in a region was calculated as the percentage of area positive for $\beta$-tubulin III staining using ImageJ. To quantitate the number of CD11c-, MHC class II-, and/ or CNTF-positive cells, images were analyzed by threshold-based automatic particle counting (http://imagej.net/Particle_Analysis).

Determination of corneal epithelial wound healing. Topical proparacaine $0.5 \%$ (Vakorn) was applied to NL and DM mice. A 2-mm circular wound was first demarcated with a trephine in the central cornea, and the epithelium was then removed with a blunt scalpel blade under a dis- 
Table 1. Primers used for PCR

\begin{tabular}{|c|c|c|}
\hline Gene & Forward primer & Reverse primer \\
\hline Bdnf & TTGTTTTGTGCCGTTTACCA & GGTAAGAGAGCCAGCCACTG \\
\hline Cntf & TCATCCCAGGAAGACTTTCG & ACAGAAGCAGCCATCTCGTT \\
\hline Nt3 & GATCCAGGCGGATATCTTGA & AGCGTCTCTGTTGCCGTAGT \\
\hline $\mathrm{Ngf}$ & САССССАСТСТСАAСAGGAT & ACCACAGCCACACACATCAA \\
\hline $\mathrm{Nt} 4 / 5$ & CCCAAGTTGAGGGAAAACAA & TССTCСGGGACAACTCСTAT \\
\hline$C d 11 c$ & GCСтTTCTTCTCCTGTTGGGG & CCAGGAAGGGTTGGTGGCAGC \\
\hline Ctgf & GCGGTGAGTCCTTCCAAAGC & GTCTTCACACTGGTGCAGCC \\
\hline$\beta$-Actin & GACGGCCAGGTCATCACTATTG & AGGAAGGCTCGAAAAGAGCC \\
\hline
\end{tabular}

secting microscope (Zeiss). Bacitracin ophthalmic ointment (Fougera) was applied to the cornea after surgery to prevent infection. To document the healing process, the remaining denuded area was visualized with fluorescein staining, photographed, and quantitated (Adobe Photoshop, version 6). The healing rate was calculated as follows: (original wound area - current wound area)/percentage of original wound area.

Subconjunctival injection of molecular reagents. Subconjunctival injection is a routine procedure used in the ophthalmology clinic to treat ocular diseases, as it allows injected materials to slowly diffuse into the cornea. The subconjunctival injection volume for mice was $5 \mu \mathrm{l}$ at 1 site. The injected concentration for Dharmacon siRNAs (GE Healthcare Dharmacon) was previously determined $(20 \mu \mathrm{M})$ (33). Dosages for neutralizing Abs, recombinant CNTF, and CNTFR $\alpha$ were determined according to the $\mathrm{ED}_{50}$ (median $\mathrm{ED}_{50}$ ) provided by the supplier, and the maximal concentrations were chosen. Anesthetized mice were injected with $5 \mu \mathrm{l} /$ cornea CNTF-neutralizing Abs (AB-557-NA; R\&D Systems; $100 \mathrm{ng} / \mu \mathrm{l}$ ) or recombinant CNTF (257-NT-010; R\&D Systems; $10 \mathrm{ng} / \mu \mathrm{l}$ ) using a 34-gauge needle and a 0.01-ml Nanofil Syringe 4-6 hours before wounding, with isoform-matched IgG or BSA as controls. To determine the function of CNTFR $\alpha$, CNTFR $\alpha$-neutralized Ab (clone AN-D3; Antibodies Online; $100 \mathrm{ng} / \mu \mathrm{l}$ ) or recombinant CNTFR $\alpha$ (558-CR; R\&D Systems; $200 \mathrm{ng} / \mu \mathrm{l}$ ) was subconjunctivally injected for 10 days every 2 days into UW corneas or 4 hours before epithelial debridement. CNTFR $\alpha$ or control siRNA was subconjunctivally injected for 10 days every 2 days into UW corneas or 24 and 4 hours beforehand into wounded corneas.

Corneal epithelial debridement wound and ocular surface staining. To study $\mathrm{SN}$ regeneration, an epithelial debridement wound was created at the center of the cornea as described previously $(8,18,23)$, resulting in the removal of epithelial nerve fibers and endings; nerve regeneration trailed behind the healing epithelial sheet (9). The wounds were allowed to heal for the indicated durations (Figure 6); the remaining wound was assessed using ocular surface fluorescence staining.

RNA extraction and PCR analyses. To determine the expression of CNTF and other neurotrophins in healing corneas, RT-PCR and qPCR was performed. For RNA isolation from epithelial cells scraped off the cornea, cDNA synthesis was performed using an oligo(dT) primer. RT-PCR and qPCR with Power SYBR Green PCR Master Mix (AB Applied Biosystems) based on $\beta$-actin expression was performed as previously described (68). Table 1 lists the primer pairs used.

Flow cytometric analysis. Flow cytometry was used to quantitate CD11c- and MHC class II-positive cells. Whole corneas were digested in $20 \mu \mathrm{l}$ Liberase TL $(2.5 \mathrm{mg} / \mathrm{ml}$; Sigma-Aldrich), followed by incu- bation at $37^{\circ} \mathrm{C}$ for 20 minutes. Cell suspensions were passed through a $70-\mu \mathrm{m}$ filter. Viable cells were then counted using trypan blue dye exclusion. A total of 5 corneas per group were examined. Cells were incubated at $4^{\circ} \mathrm{C}$ in PBS containing 2\% FBS and Fc. The cells were subsequently labeled with Cy7-conjugated CD11c and phycoerythrinMHC class II (PE-MHC class II) (clones N418 and NIMR-4, respectively; eBioscience) for 30 minutes in $2 \% \mathrm{FBS}$ at $4^{\circ} \mathrm{C}$ in the dark. All samples were washed and reconstituted in PBS. Flow cytometry was performed with a FACS system (BD FACSAria II), and the data were analyzed using FlowJo software. The numbers of CD11c- and MHC class II-positive cells were calculated from the percentage of each cell population and the precounted total number of viable cells.

Depletion of DCs. B6-DTR mice, with WT B6 mice as controls, were depleted of their DCs using a subconjunctival injection of $5 \mathrm{ng}$ DT (Sigma-Aldrich) in $5 \mu$ PBS 24 hours before wounding. Previous studies showed complete depletion of DCs locally in the cornea (33).

Statistics. Statistical analyses were performed using GraphPad Prism 6 (GraphPad Software). Data are presented as the mean \pm SD. Experiments with only 2 treatment or condition groups were analyzed for statistical significance using a 2-tailed, unpaired Student's $t$ test (Figures 1-4, Figure 5D, Figure 8, and Supplemental Figures 1 and 2). Experiments with more than 2 groups were analyzed using data calculated by 2-way ANOVA (Figure 5A and Figures 6, 7, and 9) to determine overall differences, and a Bonferroni's post test was performed to determine statistically significant differences between treatment groups. Significance was accepted at a $P$ values of less than 0.05 . Experiments were repeated at least twice to ensure reproducibility.

Study approval. The animals were housed at the Wayne State University animal care facility, which is approved by the American Association for Accreditation of Laboratory Animal Care. All experimental and animal care protocols were approved by the IACUC of Wayne State University. Weight and blood glucose levels were measured once a week after STZ injection. All investigations conformed to the guidelines of the Association for Research in Vision and Ophthalmology (ARVO) Statement on the Use of Animals in Ophthalmic and Vision Research, the NIH, and the Animal Investigation Committee of Wayne State University.

\section{Author contributions}

NG contributed to the study design, analysis and acquisition of data, and critical review of the manuscript. CY and PL contributed to the acquisition of data and reviewed the manuscript. HS contributed to the maintenance of the diabetic mice and data acquisition. FSY designed and supervised the study, drafted and reviewed the manuscript, and is responsible for the integrity of the work as a whole.

\section{Acknowledgments}

We acknowledge support from the National Eye Institute (NEI), NIH (R01EY010869 and EY017960, to F.S. Yu and p30 EY04078, to the NEI core at Wayne State University) and from Research to Prevent Blindness (to the Kresge Eye Institute). The funders had no role in study design, data collection and analysis, decision to publish, or preparation of the manuscript. We thank Dr. Qing-Sheng Mi of Henry Ford Health System and his laboratory members for their assistance with flow cytometric analysis of DCs in the cornea. 
Address correspondence to: Fu-Shin X. Yu, Kresge Eye Institute, Wayne State University School of Medicine, 4717 St. Antoine Blvd,
Detroit, Michigan 48201, USA. Phone: 313.577.1657; E-mail: fyu@ med.wayne.edu.
1. Rozsa AJ, Beuerman RW. Density and organization of free nerve endings in the corneal epithelium of the rabbit. Pain.1982;14(2):105-120.

2. Muller LJ, Marfurt CF, Kruse F, Tervo TM. Corneal nerves: structure, contents and function. Exp Eye Res. 2003;76(5):521-542.

3. Tavakoli M, Petropoulos IN, Malik RA. Assessing corneal nerve structure and function in diabetic neuropathy. Clin Exp Optom. 2012;95(3):338-347.

4. Abdelkader H, Patel DV, McGhee C, Alany RG. New therapeutic approaches in the treatment of diabetic keratopathy: a review. Clin Experiment Ophthalmol. 2011;39(3):259-270.

5. Okada Y, Reinach PS, Kitano A, Shirai K, Kao WW, Saika S. Neurotrophic keratopathy; its pathophysiology and treatment. Histol Histopathol. 2010;25(6):771-780.

6. Sacchetti M, Lambiase A. Diagnosis and management of neurotrophic keratitis. Clin Ophthalmol. 2014;8:571-579.

7. Bikbova G, Oshitari T, Tawada A, Yamamoto S. Corneal changes in diabetes mellitus. Current diabetes reviews. 2012;8(4):294-302.

8. Yin J, Huang J, Chen C, Gao N, Wang F, Yu FS. Corneal complications in streptozocin-induced type I diabetic rats. Invest Ophthalmol Vis Sci. 2011;52(9):6589-6596.

9. Wang F, Gao N, Yin J, Yu FS. Reduced innervation and delayed re-innervation after epithelial wounding in type 2 diabetic Goto-Kakizaki rats. Am J Pathol. 2012;181(6):2058-2066.

10. Lockwood A, Hope-Ross M, Chell P. Neurotrophic keratopathy and diabetes mellitus. Eye (Lond). 2006;20(7):837-839.

11. Frank RN. Diabetic retinopathy. $N$ Engl J Med. 2004;350(1):48-58.

12. Premkumar LS, Pabbidi RM. Diabetic peripheral neuropathy: role of reactive oxygen and nitrogen species. Cell Biochem Biophys. 2013;67(2):373-383.

13. Jack M, Wright D. Role of advanced glycation endproducts and glyoxalase I in diabetic peripheral sensory neuropathy. Transl Res. 2012;159(5):355-365.

14. Dobrowsky RT, Rouen S, Yu C. Altered neurotrophism in diabetic neuropathy: spelunking the caves of peripheral nerve. J Pharmacol Exp Ther. 2005;313(2):485-491.

15. Vincent AM, Callaghan BC, Smith AL, Feldman EL. Diabetic neuropathy: cellular mechanisms as therapeutic targets. Nat Rev Neurol. 2011;7(10):573-583.

16. Davidson EP, Coppey LJ, Holmes A, Yorek MA. Changes in corneal innervation and sensitivity and acetylcholine-mediated vascular relaxation of the posterior ciliary artery in a type 2 diabetic rat. Invest Ophthalmol Vis Sci. 2012;53(3):1182-1187.

17. Davidson EP, Coppey LJ, Yorek MA. Early loss of innervation of cornea epithelium in streptozotocin-induced type 1 diabetic rats: improvement with ilepatril treatment. Invest Ophthalmol Vis Sci. 2012;53(13):8067-8074.

18. Xu K, Yu FS. Impaired epithelial wound healing and EGFR signaling pathways in the corneas of diabetic rats. Invest Ophthalmol Vis Sci.

\section{1;52(6):666}

19. Tavakoli M, et al. Corneal confocal microscopy detects early nerve regeneration in diabetic neuropathy after simultaneous pancreas and kidney transplantation. Diabetes. 2013;62(1):254-260.

20. Efron N. The Glenn A. Fry award lecture 2010: Ophthalmic markers of diabetic neuropathy. Optom Vis Sci. 2011;88(6):661-683.

21. Saleh A, et al. Ciliary neurotrophic factor activates NF-kappaB to enhance mitochondrial bioenergetics and prevent neuropathy in sensory neurons of streptozotocin-induced diabetic rodents. Neuropharmacology. 2013;65:65-73.

22. Karamoysoyli E, Burnand RC, Tomlinson DR, Gardiner NJ. Neuritin mediates nerve growth factor-induced axonal regeneration and is deficient in experimental diabetic neuropathy. Diabetes. 2008;57(1):181-189.

23. Wang F, Gao N, Yin J, Yu FS. Reduced innervation and delayed re-innervation after epithelial wounding in type 2 diabetic Goto-Kakizaki rats. Am J Pathol. 2012;181(6):2058-2066.

24. Davidson EP, Coppey LJ, Kardon RH, Yorek MA. Differences and similarities in development of corneal nerve damage and peripheral neuropathy and in diet-induced obesity and type 2 diabetic rats. Invest Ophthalmol Vis Sci. 2014;55(3):1222-1230.

25. McGovern AE, Mazzone SB. Neural regulation of inflammation in the airways and lungs. Auton Neurosci. 2014;182:95-101.

26. Giusto E, Donega M, Cossetti C, Pluchino S. Neuro-immune interactions of neural stem cell transplants: from animal disease models to human trials. Exp Neurol. 2014;260:19-32.

27. Clark AK, Malcangio M. Fractalkine/CX3CR1 signaling during neuropathic pain. Front Cell Neurosci. 2014;8:121.

28. Feng P, Yee KK, Rawson NE, Feldman LM, Feldman RS, Breslin PA. Immune cells of the human peripheral taste system: dominant dendritic cells and CD4 T cells. Brain Behav Immun. 2009;23(6):760-766.

29. Veres TZ, Rochlitzer S, Braun A. The role of neuro-immune cross-talk in the regulation of inflammation and remodelling in asthma. Pharmacol Ther. 2009;122(2):203-214.

30. Veres TZ, et al. Dendritic cell-nerve clusters are sites of $\mathrm{T}$ cell proliferation in allergic airway inflammation. Am J Pathol. 2009;174(3):808-817.

31. Le DD, et al. Allergic airway inflammation induces the migration of dendritic cells into airway sensory ganglia. Respir Res. 2014;15:73.

32. Leppin K, et al. Diabetes mellitus leads to accumulation of dendritic cells and nerve fiber damage of the subbasal nerve plexus in the cornea. Invest Ophthalmol Vis Sci. 2014;55(6):3603-3615.

33. Gao N, Yin J, Yoon GS, Mi QS, Yu FS. Dendritic cell-epithelium interplay is a determinant factor for corneal epithelial wound repair. Am J Pathol. 2011;179(5):2243-2253.

34. de Jong PR, et al. TRPM8 on mucosal sensory nerves regulates colitogenic responses by innate immune cells via CGRP. Mucosal Immunol. 2014;8(3):491-504.
35. Takashima A. Harnessing DCs by substance P. Blood. 2013;121(15):2815-2816.

36. Voedisch S, Rochlitzer S, Veres TZ, Spies E, Braun A. Neuropeptides control the dynamic behavior of airway mucosal dendritic cells. PLoS One. 2012;7(9):e45951.

37. Sun H, Mi X, Gao N, Yan C, Yu FS. Hyperglycemia-suppressed expression of serpine 1 contributes to delayed epithelial wound healing in diabetic mouse corneas. Invest Ophthalmol Vis Sci. 2015;56(5):3383-3392.

38. Kallinikos P, Berhanu M, O’Donnell C, Boulton AJ, Efron N, Malik RA. Corneal nerve tortuosity in diabetic patients with neuropathy. Invest Ophthalmol Vis Sci. 2004;45(2):418-422.

39. Cai J, et al. Potential therapeutic effects of neurotrophins for acute and chronic neurological diseases. Biomed Res Int. 2014;2014:601084.

40. Oliveira SL, Pillat MM, Cheffer A, Lameu C, Schwindt TT, Ulrich H. Functions of neurotrophins and growth factors in neurogenesis and brain repair. Cytometry A. 2013;83(1):76-89.

41. Kim EC, Lee WS, Kim MS. The inhibitory effects of bevacizumab eye drops on NGF expression and corneal wound healing in rats. Invest Ophthalmol Vis Sci. 2010;51(9):4569-4573.

42. Sarkar J, et al. CD11b ${ }^{+} \mathrm{GR} 1^{+}$myeloid cells secrete NGF and promote trigeminal ganglion neurite growth: implications for corneal nerve regeneration. Invest Ophthalmol Vis Sci. 2013;54(9):5920-5936.

43. Sleeman MW, Anderson KD, Lambert PD, Yancopoulos GD, Wiegand SJ. The ciliary neurotrophic factor and its receptor, CNTFR alpha. Pharm Acta Helv. 2000;74(2-3):265-272.

44. Davis S, et al. Released form of CNTF receptor alpha component as a soluble mediator of CNTF responses. Science. 1993;259(5102):1736-1739.

45. Ozog MA, Modha G, Church J, Reilly R, Naus CC. Co-administration of ciliary neurotrophic factor with its soluble receptor protects against neuronal death and enhances neurite outgrowth. J Biol Chem . 2008;283(10):6546-6560.

46. Robledo O, et al. Binding interactions of leukemia inhibitory factor and ciliary neurotrophic factor with the different subunits of their high affinity receptors. J Neurochem. 1996;66(4):1391-1399.

47. Bar-On L, Jung S. Defining in vivo dendritic cell functions using CD11c-DTR transgenic mice. Methods Mol Biol. 2010;595:429-442.

48. Muller A, Zhang EP, Schroff M, Wittig B, Hoffmann $\mathrm{F}$. Influence of ballistic gene transfer on antigen-presenting cells in murine corneas. Graefes Arch Clin Exp Ophthalmol. 2002;240(10):851-859.

49. Kao WW, Zhu G, Benza R, Kao CW, Ishizaki M, Wander AH. Appearance of immune cells and expression of MHC II DQ molecule by fibroblasts in alkali-burned corneas. Cornea. 1996;15(4):397-408.

50. Strom A, et al. Pronounced reduction of cutaneous Langerhans cell density in recently diagnosed type 2 diabetes. Diabetes. 2014;63(3):1148-1153.

51. Stojadinovic O, Yin N, Lehmann J, Pastar I, Kirs- 
ner RS, Tomic-Canic M. Increased number of Langerhans cells in the epidermis of diabetic foot ulcers correlates with healing outcome. Immunol Res. 2013;57(1-3):222-228.

52. Yan C, et al. Targeting imbalance between interleukin-1 $\beta$ and IL-1 receptor antagonist ameliorates delayed epithelium wound healing in diabetic mouse corneas. Ame J Pathol. 2016:in press.

53. Soloff AC, et al. Massive mobilization of dendritic cells during influenza A virus subtype H5N1 infection of nonhuman primates. J Infect Dis. 2014;209(12):2012-2016.

54. Lee N, et al. Muscle ciliary neurotrophic factor receptor alpha promotes axonal regeneration and functional recovery following peripheral nerve lesion. J Comp Neurol. 2013;521(13):2947-2965.

55. Wen R, Tao W, Li Y, Sieving PA. CNTF and retina. Prog Retin Eye Res. 2012;31(2):136-151.

56. Reichard M, Hovakimyan M, Guthoff RF, Stachs O. In vivo visualisation of murine corneal nerve fibre regeneration in response to ciliary neurotrophic factor. Exp Eye Res. 2014;120:20-27.

57. Chung ES, et al. Expression of neurotrophic factors and their receptors in keratoconic cornea. Curr Eye Res. 2013;38(7):743-750.
58. Qi H, et al. Patterned expression of neurotrophic factors and receptors in human limbal and corneal regions. Mol Vis. 2007;13:1934-1941.

59. Zhou Q, et al. Ciliary neurotrophic factor promotes the activation of corneal epithelial stem/ progenitor cells and accelerates corneal epithelial wound healing. Stem Cells. 2015;33(5):1566-1576.

60. Zhou Q, et al. Ciliary neurotrophic factor promotes the activation of corneal epithelial stem/ progenitor cells and accelerates corneal epithelial wound healing. Stem Cells. 2014;33(5):1566-1576.

61. Pasquin S, Sharma M, Gauchat JF. Ciliary neurotrophic factor (CNTF): New facets of an old molecule for treating neurodegenerative metabolic syndrome pathologies. Cytokine Growth Factor Rev. 2015;26(5):507-515.

62. Schuster B, et al. Signaling of human ciliary neurotrophic factor (CNTF) revisited. The interleukin-6 receptor can serve as an alpha-receptor for CTNF. J Biol Chem. 2003;278(11):9528-9535.

63. Skundric DS, Lisak RP. Role of neuropoietic cytokines in development and progression of diabetic polyneuropathy: from glucose metabolism to neurodegeneration. Exp Diabesity Res. 2003;4(4):303-312.
64. Rhee KD, Nusinowitz S, Chao K, Yu F, Bok D, Yang XJ. CNTF-mediated protection of photoreceptors requires initial activation of the cytokine receptor gp130 in Muller glial cells. Proc Natl Acad Sci U S A. 2013;110(47):E4520-E4529.

65. Leung E, Landa G. Update on current and future novel therapies for dry age-related macular degeneration. Exp Rev Clin Pharmacol. 2013;6(5):565-579.

66. Zhang K, et al. Ciliary neurotrophic factor delivered by encapsulated cell intraocular implants for treatment of geographic atrophy in age-related macular degeneration. Proc Natl Acad Sci U S A. 2011;108(15):6241-6245.

67. Xu KP, Li Y, Ljubimov AV, Yu FS. High glucose suppresses epidermal growth factor receptor/ phosphatidylinositol 3-kinase/Akt signaling pathway and attenuates corneal epithelial wound healing. Diabetes. 2009;58(5):1077-1085.

68. Bettahi I, et al. Genome-wide transcriptional analysis of differentially expressed genes in diabetic, healing corneal epithelial cells: hyperglycemia-suppressed TGF $\beta 3$ expression contributes to the delay of epithelial wound healing in diabetic corneas. Diabetes. 2014;63(2):715-727. 